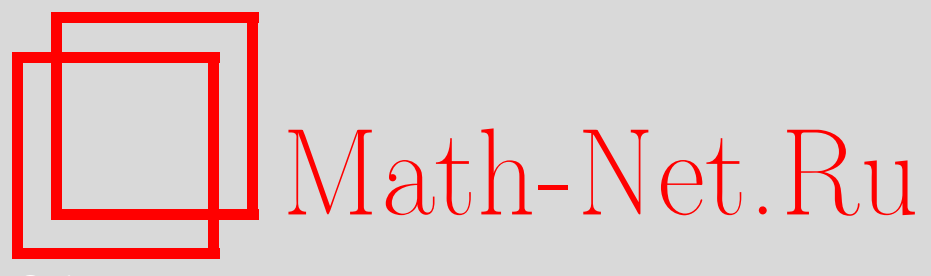

В. А. Мирзоян, Классификация одного класса минимальных полуэйнштейновых подмногообразий с интегрируемым распределением кодефектности, Матем. сб., 2008, том 199, номер 3, 69-94

DOI: https://doi.org/10.4213/sm3778

Использование Общероссийского математического портала Math-Net.Ru подразумевает, что вы прочитали и согласны с пользовательским соглашением http: //www . mathnet.ru/rus/agreement

Параметры загрузки:

IP: 54.147 .182 .235

26 апреля 2023 г., 12:34:53

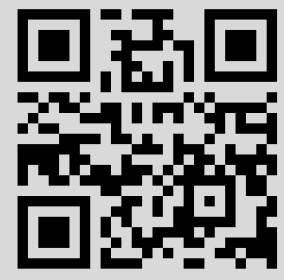




\author{
В. А. Мирзоян
}

\title{
Классификация одного класса минимальных полуэйнштейновых подмногообразий с интегрируемым распределением кодефектности
}

\begin{abstract}
В евклидовых пространствах дается полная локальная классификация и геометрическое описание нормально плоских минимальных полуэйнштейновых подмногообразий с кратными главными векторами кривизны и интегрируемым распределением кодефектности.

Библиография: 30 названий.
\end{abstract}

\section{§ 1. Формулировка результатов}

Римановы Ric-полусимметрические пространства характеризуются условием полупараллельности тензора Риччи $R_{1}, R(X, Y) \cdot R_{1}=0$, и являются естественными обобщениями римановых полусимметрических пространств (характеризуемых условием $R(X, Y) \cdot R=0$, где $R$ - тензор кривизны), римановых пространств с параллельным тензором Риччи $\left(\nabla R_{1}=0\right)$, эйнштейновых и симметрических пространств. В этой связи Ric-полусимметрические пространства и их изометрические погружения стали предметом исследования многих авторов (см. обзор [1] и цитированную там литературу).

В [2] автором было доказано, что риманово пространство $M$ удовлетворяет условию $R(X, Y) \cdot R_{1}=0$ тогда и только тогда, когда оно является либо двумерным, либо эйнштейновым, либо полуэйнштейновым, либо прямым произведением таких пространств.

Полуэйнштейновы пространства определяются следующим образом. Пусть $T_{x}^{(0)}$ обозначает пространство дефектности тензора кривизны риманова пространства $M$ в точке $x$, а $T_{x}^{(1)}$ - его ортогональное дополнение в касательном пространстве $T_{x}(M)$, называемое пространством кодефектности в этой точке. Соответствующие распределения $T^{(0)}$ и $T^{(1)}$ называются распределениями дефектности и кодефектности. Пространства $T_{x}^{(0)}$ и $T_{x}^{(1)}$ инвариантны относительно тензора Риччи $R_{1}$, и справедливо следующее разложение в прямую сумму:

$$
T_{x}(M)=T_{x}^{(0)}+T_{x}^{(1)} .
$$

Размерности

$$
\mu_{x}=\operatorname{dim} T_{x}^{(0)}, \quad \operatorname{dim} T_{x}^{(1)}=\operatorname{dim} T_{x}(M)-\mu_{x}
$$

называются соответственно индексами дефектности и кодефектности пространства $M$ в точке $x$. В дальнейшем будем предполагать, что индекс $\mu_{x}$ является локально постоянным, т.е. $\mu_{x}=\mu$.

Риманово пространство $M$ называется полуэйнштейновым, если в каждой точке $x$ тензор Риччи $R_{1}$ имеет на $T_{x}^{(1)}$ только одно ненулевое собственное 
значение (см. [2]). Примерами полуэйнштейновых пространств служат конусы над эйнштейновыми пространствами (см. [3], [4]) и римановы многообразия кодефектности два, которые всесторонне исследовались в [5]. В евклидовом пространстве $E_{n}$ примеры полуэйнштейновых подмногообразий, имеющих вид конусов над эйнштейновыми пространствами, отличными от пространств постоянной кривизны, получены автором в [4] и [6] (в работе [6] в одном случае это сделано при неявном предположении интегрируемости распределения кодефектности подмногообразия) и Ф. Дефевером (см. [7]).

Настоящая работа посвящена классификации и геометрическому описанию одного класса нормально плоских минимальных полуэйнштейновых подмногообразий с интегрируемым распределением кодефектности. Основной результат выражается следующей теоремой.

Теорема 1. Пусть в евклидовом пространстве $E_{n}$ m-мерное нормально плоское минимальное полуэйнштейново подмногообразие $M$ индекса дефектности $\mu \geqslant 1$ имеет в каждой точке $x \in M$ только $q \geqslant 2$ различных ненулевых главных векторов кривизны $n_{1}, \ldots, n_{q}$ с кратностями $p_{1} \geqslant 2, \ldots, p_{q} \geqslant 2$ соответственно. Если распределение кодефектности $T^{(1)}$ подмногообразия $M$ является интегрируемым с интегральным многообразием $M^{(1)}$, то $M$ локально либо представляет собой цилиндр над $M^{(1)}$, m.е. $M=E_{\mu} \times M^{(1)}$, где $E_{\mu}-$ плоскость размерности $\mu$, либо имеет вид прямого произведения $E_{\mu-1} \times \widetilde{M}$, где $E_{\mu-1}$ - плоскость размерности $\mu-1$, а $\widetilde{M}$ является конусом над $M^{(1)}$, причем в последнем случае $M^{(1)}$ принадлежит некоторой гиперсфере пространства $E_{n}$ и является минимальным в этой гиперсфере.

В обоих случаях $M^{(1)}$ несет ортогональную сопряженную систему, состоящую из q сфер $S^{p_{1}}(R), \ldots, S^{p_{q}}(R)$ и (как подмногообразие в $\left.E_{n}\right)$ имеет плоскую нормальную связность и является эйнштейновым.

Более того, в случае иилиндра $M^{(1)}$ либо представляет собой скрученное произведение указанных сфер, либо является прямым произведением минимальных скрученных произведений различных групп этих сфер.

В случае конуса в зависимости от значения $q$ подмногообразие $M^{(1)}$ характеризуется следующим образом:

(1) при $q=2 M^{(1)}$ есть прямое произведение сфер $S^{p_{1}}(R), S^{p_{2}}(R)$ и, кроме mого, $p_{1}=p_{2}$;

(2) $п р и q \geqslant 3 M^{(1)}$ является либо (а) прямым произведением сфер $S^{p_{1}}(R)$, $\ldots, S^{p_{q}}(R)$, причем в этом случае $p_{1}=\cdots=p_{q}$, либо (б) скрученным произведением этих сфер, либо (в) прямым произведением вида $N_{0} \times N_{1} \times \cdots \times N_{\tau}$, где $N_{0}$ есть прямое произведение некоторых из этих сфер, а $N_{1}, \ldots, N_{\tau}-$ скрученные произведения различных групп остальных сфер.

В теореме 1 под скрученным произведением сфер имеется в виду такое неприводимое подмногообразие $M$ в $E_{n}$, которое локально несет ортогональную сопряженную систему из $q \geqslant 3$ сфер, вращающихся друг относительно друга при движении вдоль этого подмногообразия.

Отметим, что описываемое в теореме 1 подмногообразие $M^{(1)}$ представляет определенный интерес как эйнштейново подмногообразие коразмерности $\geqslant 2$. Тензор Риччи цилиндра $E_{\mu} \times M^{(1)}$ является параллельным. Тензор Риччи произведения $E_{\mu-1} \times \widetilde{M}$ является полупараллельным и не удовлетворяет более 
сильному условию. Скрученные произведения сфер специального вида ранее были получены в [8], [9] при описании полусимметрических подмногообразий. Скрученные произведения более общего вида рассматривались в [10], [11]. Отметим также, что в последнее время условия $R(X, Y) \cdot R=0$ и $R(X, Y) \cdot R_{1}=0$ и некоторые их обобщения интенсивно исследуются в полуримановой геометрии и в теории различных классов комплексных и контактных многообразий (см. работы [12]-[18] и цитированную в них литературу).

На протяжении всей статьи мы будем использовать различные понятия, известные результаты и стандартные формулы римановой геометрии, которые можно найти в [19]-[24]. С теорией минимальных подмногообразий можно ознакомиться в известных монографиях [25], [26].

\section{§ 2. Аппарат исследования и редукция некоторых условий}

Пусть $O\left(E_{n}\right)$ является главным расслоением ортонормированных реперов $\left\{x, e_{1}, \ldots, e_{n}\right\}$ в евклидовом пространстве $E_{n}$. Отождествляя точку $x$ с ее радиус-вектором, будем иметь

$$
d x=\omega^{A} e_{A}, \quad d e_{A}=\omega_{A}^{B} e_{B}, \quad \omega_{B}^{A}+\omega_{A}^{B}=0, \quad A, B, \ldots=1, \ldots, n .
$$

Отсюда внешним дифференцированием получаются следующие структурные уравнения:

$$
d \omega^{A}=\omega^{B} \wedge \omega_{B}^{A}, \quad d \omega_{A}^{B}=\omega_{A}^{C} \wedge \omega_{C}^{B} .
$$

Пусть $M$ является $m$-мерным подмногообразием в $E_{n}$. Тогда расслоение $O\left(E_{n}\right)$ может быть приведено к главному расслоению $O\left(M, E_{n}\right)$ адаптированных к $M$ ортонормированных реперов $\left\{x, e_{1}, \ldots, e_{m}, e_{m+1}, \ldots, e_{n}\right\}$, характеризуемых тем, что $x \in M$, векторы $e_{i}, i, j, \ldots=1, \ldots, m$, принадлежат касательному пространству $T_{x}(M)$, а векторы $e_{\alpha}, \alpha, \beta, \ldots=m+1, \ldots, n$, принадлежат нормальному пространству $T_{x}^{\perp}(M)$. В силу этого по известной схеме, приведенной, например, в [8], [22], [24], можем прийти к следующим соотношениям:

$$
\begin{gathered}
\omega^{\alpha}=0, \quad \omega_{i}^{\alpha}=h_{i j}^{\alpha} \omega^{j}, \quad h_{i j}^{\alpha}=h_{j i}^{\alpha}, \\
d h_{i j}^{\alpha}+h_{i j}^{\beta} \omega_{\beta}^{\alpha}-h_{k j}^{\alpha} \omega_{i}^{k}-h_{i k}^{\alpha} \omega_{j}^{k}=h_{i j k}^{\alpha} \omega^{k}, \quad h_{i j k}^{\alpha}=h_{i k j}^{\alpha} .
\end{gathered}
$$

Здесь функции $h_{i j}^{\alpha}, h_{i j k}^{\alpha}$, симметричные по нижним индексам, являются компонентами второй и третьей фундаментальных форм $\alpha_{2}$ и $\alpha_{3}$ соответственно. Действие формы $\alpha_{2}$ определяется следующим образом: если $X=X^{i} e_{i}$, $Y=Y^{j} e_{j}-$ произвольные касательные к подмногообразию $M$ векторы, то $\alpha_{2}(X, Y)=h_{i j}^{\alpha} X^{i} Y^{j} e_{\alpha}$. Аналогично определяется действие формы $\alpha_{3}$.

Если $\alpha_{2}=0$, т.е. $h_{i j}^{\alpha}=0$, то подмногообразие называется вполне геодезическим. Если $h_{i j}^{\alpha}=\lambda^{\alpha} \delta_{i j}$ для любого значения $\alpha$, то подмногообразие называется вполне омбилическим. Инвариантно определенный нормальный вектор $H=H^{\alpha} e_{\alpha}$, где $H^{\alpha}=\sum_{i} h_{i i}^{\alpha}$ (в целях удобства коэффициент $1 / m$ опускаем), называется вектором средней кривизны подмногообразия. Если $H=0$, то подмногообразие называется минимальным. Индексом относительной дефектноcmu подмногообразия $M$ в точке $x$ называется размерность подпространства $T_{x}^{\prime}$ касательного пространства $T_{x}(M)$, определяемого равенством

$$
T_{x}^{\prime}=\left\{X \in T_{x}(M) ; \alpha_{2}(X, Y)=0 \forall Y \in T_{x}(M)\right\}
$$


(см. [27]).

В соотношении (2.2) 1-формы $\omega_{j}^{i}$ определяют на подмногообразии $M$ риманову связность $\nabla$, а 1-формы $\omega_{\beta}^{\alpha}$ определяют связность в нормальном расслоении $T^{\perp}(M)$, которая называется нормальной связностъю и обозначается через $\nabla^{\perp}$. Формы кривизны этих связностей определяются равенствами

$$
\begin{aligned}
& \Omega_{i}^{j}=d \omega_{i}^{j}-\omega_{i}^{k} \wedge \omega_{k}^{j}=-\sum_{\alpha} h_{i[k}^{\alpha} h_{l] j}^{\alpha} \omega^{k} \wedge \omega^{l}, \\
& \Omega_{\alpha}^{\beta}=d \omega_{\alpha}^{\beta}-\omega_{\alpha}^{\gamma} \wedge \omega_{\gamma}^{\beta}=-\sum_{i} h_{i[k}^{\alpha} h_{l] i}^{\beta} \omega^{k} \wedge \omega^{l},
\end{aligned}
$$

а их коэффициенты

$$
R_{i k l}^{j}=-\sum_{\alpha} h_{i[k}^{\alpha} h_{l] j}^{\alpha}, \quad R_{\alpha k l}^{\beta}=-\sum_{i} h_{i[k}^{\alpha} h_{l] i}^{\beta}
$$

представляют собой компоненты тензоров кривизны $R$ и $R^{\perp}$ связностей $\nabla$ и $\nabla^{\perp}$ соответственно. Действие тензора $R$ определяется следующим образом: если

$$
X=X^{i} e_{i}, \quad Y=Y^{j} e_{j}, \quad Z=Z^{k} e_{k},
$$

Tо

$$
R(X, Y) Z=R_{i j k}^{l} X^{i} Y^{j} Z^{k} e_{l} .
$$

Напомним, что пространство дефектности $T_{x}^{(0)}$ в точке $x \in M$ определяется следующим равенством:

$$
T_{x}^{(0)}=\left\{X \in T_{x}(M) ; R(X, Y)=0 \forall Y \in T_{x}(M)\right\} .
$$

Справедливо включение $T_{x}^{\prime} \subset T_{x}^{(0)}$ (см. [27]). Отметим также, что $T_{x}^{(0)}$ всегда содержится в собственном подпространстве тензора Риччи $R_{1}$, отвечающем нулевому собственному значению (см., например, [2]). Если $R=0$, то подмногообразие называется локально евклидовым. При $R^{\perp}=0$ нормальная связность называется плоской, а само подмногообразие - нормалъно плоским. Компоненты $R_{i k}$ тензора Риччи $R_{1}$ определяются по формуле

$$
R_{i k}=R_{i k l}^{l}=\sum_{\alpha}\left(h_{i l}^{\alpha} h_{k}^{\alpha l}-H^{\alpha} h_{i k}^{\alpha}\right),
$$

где $H^{\alpha}$ - компоненты вектора средней кривизны $H$.

Пусть нормальная связность подмногообразия $M$ является плоской, т.е. $R_{\alpha k l}^{\beta}=0$. В этом случае все матрицы $\left\|h_{i j}^{\alpha}\right\|$ коммутируют между собой, и в силу этого в некотором ортонормированном репере они могут быть одновременно приведены к диагональному виду $\left\|\lambda_{i}^{\alpha} \delta_{i j}\right\|$. Подставляя в формулу для компонент $R_{i k}$ тензора Риччи $R_{1}$, получим

$$
R_{i k}=\sum_{\alpha}\left[\left(\lambda_{i}^{\alpha}\right)^{2}-H^{\alpha} \lambda_{i}^{\alpha}\right] \delta_{i k} .
$$

Отсюда следует, что матрица $\left\|R_{i k}\right\|$ имеет диагональный вид с диагональными элементами

$$
\rho_{i}=\sum_{\alpha}\left[\left(\lambda_{i}^{\alpha}\right)^{2}-H^{\alpha} \lambda_{i}^{\alpha}\right] .
$$


Нормальные векторы $n_{i}=\lambda_{i}^{\alpha} e_{\alpha}$ называются главными векторами кривизны нормально плоского подмногообразия. Легко видеть, что $H=n_{1}+\cdots+n_{m}$. Если $\langle$,$\rangle - скалярное произведение в E_{n}$, то

$$
\sum_{\alpha}\left(\lambda_{j}^{\alpha}\right)^{2}=\left\langle n_{j}, n_{j}\right\rangle=\left|n_{j}\right|^{2}, \quad \sum_{\alpha} \lambda_{j}^{\alpha} \lambda_{k}^{\alpha}=\left\langle n_{j}, n_{k}\right\rangle,
$$

где вторая сумма есть секционная кривизна в направлении $e_{j} \wedge e_{k}$.

Пусть подмногообразие $M$ является нормально плоским и минимальным, т.е. $H=0$. Тогда из $(2.3)$ следует, что $\rho_{i}=\left|n_{j}\right|^{2}$. На основании этого равенства можем сформулировать следующие утверждения:

а) в евклидовом пространстве $E_{n}$ нормально плоское минимальное подмногообразие $M$ является полуэйнштейновым тогда и толъко тогда, когда его ненулевые главные векторы кривизны имеют равные модули;

б) в евклидовом пространстве $E_{n}$ индекс дефектности нормально плоского минимального подмногообразия в каждой точке равен индексу его относительной дефектности (подробности доказательства см. в [28]).

Итак, пусть $M$ является $m$-мерным нормально плоским минимальным полуэйнштейновым подмногообразием индекса дефектности $\mu$ в $E_{n}$, и пусть $M$ имеет $q$ ненулевых главных векторов кривизны $n_{1}, \ldots, n_{q}$ с кратностями $p_{1}, \ldots, p_{q}$ соответственно, $p_{1}+\cdots+p_{q}=m-\mu$. Условия минимальности и полуэйнштейновости равносильны соответственно следующим условиям:

$$
p_{1} n_{1}+\cdots+p_{q} n_{q}=0, \quad\left|n_{1}\right|^{2}=\cdots=\left|n_{q}\right|^{2}=\rho \neq 0 .
$$

Из первого равенства в (2.4) следует, что векторы $n_{1}, \ldots, n_{q}$ линейно зависимы. Если $\operatorname{rang}\left(n_{1}, \ldots, n_{q}\right)=\delta$, то $\delta \leqslant n-m$ или $\mu+p_{1}+\cdots+p_{q}+\delta \leqslant n$. Если $\delta$ достигает своего наибольшего значения $q-1$, то $\mu+p_{1}+\cdots+p_{q}+q \leqslant n+1$. Из условий (2.4) следует также, что при $q \geqslant 3$ кратности $p_{1}, \ldots, p_{q}$ "составляют" $q$-угольник с целочисленными сторонами, который расположен в $\delta$-мерной плоскости. Следовательно, система чисел $p_{1}, \ldots, p_{q}$ обладает таким свойством: сумма любых $q-1$ чисел этой системы больше оставшегося $q$-го числа (это доказано ниже также аналитически).

Пусть $\theta_{\varphi \psi}, \varphi, \psi=1, \ldots, q$ обозначает угол между векторами $n_{\varphi}$ и $n_{\psi}$. Умножая последовательно первое из равенств (2.4) скалярно на $n_{1}, \ldots, n_{q}$ и сокращая на $\rho$, получим следующую систему:

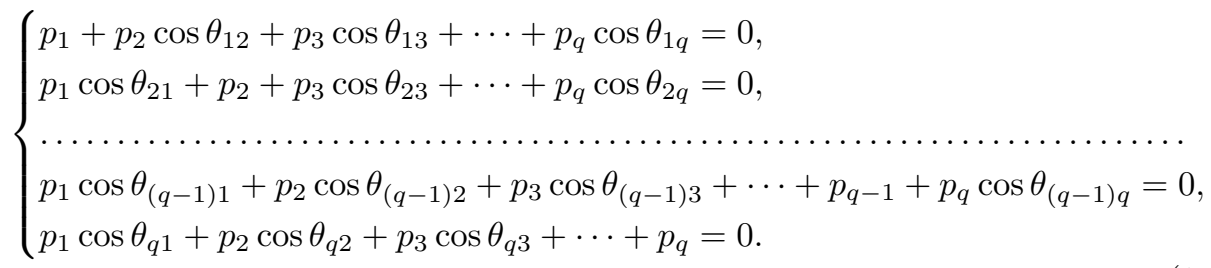

Если, например, из первых $q-1$ уравнений этой системы определить $\cos \theta_{1 q}$, $\cos \theta_{2 q}, \ldots, \cos \theta_{(q-1) q}$ и подставить в последнее уравнение, то получим

$$
p_{1}^{2}+\cdots+p_{q-1}^{2}+2 p_{1} p_{2} \cos \theta_{12}+\cdots+2 p_{q-2} p_{q-1} \cos \theta_{(q-2)(q-1)}=p_{q}^{2} .
$$

Тогда

$$
p_{1}^{2}+\cdots+p_{q-1}^{2}+2 p_{1} p_{2}+\cdots+2 p_{q-2} p_{q-1}>p_{q}^{2}
$$


и, следовательно,

$$
p_{1}+\cdots+p_{q-1}>p_{q}
$$

Пусть в (2.5) все углы равны между собой, т.е. $\theta_{\varphi \psi}=\theta$ для любых неравных значений индексов. Тогда (2.5) принимает следующий вид:

$$
\left\{\begin{array}{l}
p_{1}+\left(p_{2}+p_{3}+\cdots+p_{q}\right) \cos \theta=0, \\
p_{2}+\left(p_{1}+p_{3}+\cdots+p_{q}\right) \cos \theta=0, \\
\left.\cdots \cdots \cdots \cdots \cdots \cdots \cdots \cdots \cdots \cdots \cdots \cdots \cdots \cdots+p_{q-1}\right) \cos \theta=0 \\
p_{q}+\left(p_{1}+p_{2}+\cdots \cdots+\right.
\end{array}\right.
$$

Исключая из первых двух уравнений этой системы $\cos \theta$, получим

$$
\left(p_{2}-p_{1}\right)\left(p_{1}+p_{2}+\cdots+p_{q}\right)=0 .
$$

Следовательно, $p_{1}=p_{2}$. Точно так же доказывается, что $p_{2}=p_{3}=\cdots=p_{q}$. При $q=3$ и $p_{1}=p_{2}=p_{3}$ из системы (2.5) легко получить, что $\theta_{12}=\theta_{13}=$ $\theta_{23}=120^{\circ}$. При $q \geqslant 4$ равенство кратностей $p_{1}, \ldots, p_{q}$, вообще говоря, не влечет равенство углов $\theta_{\varphi \psi}$. Отметим, что при равенстве всех углов $\theta_{\varphi \psi}$ число $q$ главных векторов кривизны может достигать своего максимального значения $n-m+1$ (подробности см. в [28]).

\section{§ 3. Доказательство основных лемм}

В настоящем параграфе мы выявим рад важнейших свойств нормально плоского минимального полуэйнштейнова подмногообразия в одном частном случае и получим значительное представление о его локальной структуре.

Пусть $M$ является $m$-мерным нормально плоским минимальным полуэйнштейновым подмногообразием индекса дефектности $\mu \geqslant 1$ в евклидовом пространстве $E_{n}, n-m \geqslant 2$. Пусть в каждой точке $x \in M$ имеется $q \geqslant 2$ различных ненулевых главных векторов кривизны $n_{1}, \ldots, n_{q}$ кратностей $p_{1} \geqslant 2, \ldots$, $p_{q} \geqslant 2$ соответственно и однократные ненулевые главные векторы кривизны $n_{q+1}, \ldots, n_{q+q_{1}}$. Нулевой главный вектор кривизны имеет, как мы знаем, кратность $\mu$. Условия минимальности и полуэйнштейновости подмногообразия $M$ (см. (2.4)) принимают соответственно вид

$$
\begin{gathered}
p_{1} n_{1}+\cdots+p_{q} n_{q}+n_{q+1}+\cdots+n_{q+q_{1}}=0, \\
\left|n_{1}\right|^{2}=\cdots=\left|n_{q}\right|^{2}=\left|n_{q+1}\right|^{2}=\cdots=\left|n_{q+q_{1}}\right|^{2}=\rho .
\end{gathered}
$$

Условимся, что в дальнейшем индексы будут принимать следующие значения:

$$
\begin{gathered}
i, j, k=1, \ldots, m, \quad \alpha, \beta, \gamma=m+1, \ldots, n, \quad a, b, c=1, \ldots, p_{1}+\cdots+p_{q}=p \\
u, v, w=p+1, \ldots, p+q_{1}, \quad r, s, t=p+q_{1}+1, \ldots, m \\
\varphi, \psi, \chi=1, \ldots, q, \quad \varphi_{1}, \psi_{1}, \chi_{1}=q+1, \ldots, q+q_{1} \\
i_{\varphi}, j_{\varphi}, k_{\varphi}=p_{1}+\cdots+p_{\varphi-1}+1, \ldots, p_{1}+\cdots+p_{\varphi-1}+p_{\varphi} .
\end{gathered}
$$

Будем предполагать, что $p_{0}=0$ по определению. 
Поскольку вектор $n_{\varphi}$ имеет кратность $p_{\varphi}$, то будем считать, что $n_{i_{\varphi}}=$ $n_{j_{\varphi}}=n_{\varphi}$ при $i_{\varphi} \neq j_{\varphi}$. Тогда $\lambda_{i_{\varphi}}^{\alpha}=\lambda_{j_{\varphi}}^{\alpha}=\lambda_{(\varphi)}^{\alpha}$ при $i_{\varphi} \neq j_{\varphi}$, хотя в дальнейшем в случае необходимости наряду с записью $\lambda_{(\varphi)}^{\alpha}$ мы будем использовать также запись $\lambda_{a}^{\alpha}$. Так как в наших обозначениях главные векторы кривизны $n_{r}$ нулевые, то $\lambda_{r}^{\alpha}=0$. Условия минимальности и полуэйнштейновости подмногообразия $M$ равносильны соответственно следующим условиям:

$$
\sum_{\varphi} p_{\varphi} \lambda_{(\varphi)}^{\alpha}+\sum_{u} \lambda_{u}^{\alpha}=0, \quad \sum_{\alpha}\left(\lambda_{(\varphi)}^{\alpha}\right)^{2}=\sum_{\alpha}\left(\lambda_{u}^{\alpha}\right)^{2}=\rho .
$$

В принятых обозначениях матрицы $\left\|h_{i j}^{\alpha}\right\|$ второй фундаментальный формы подмногообразия $M$ принимают следующий вид:

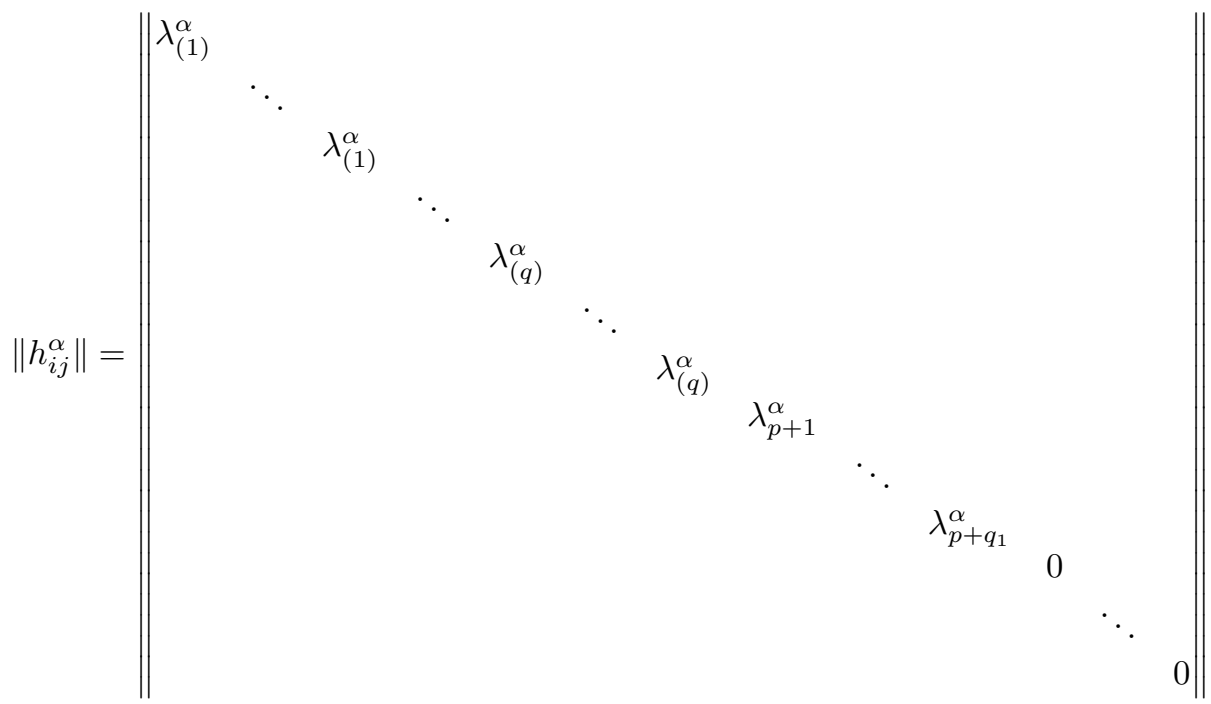

В этих матрицах $\lambda_{(\varphi)}^{\alpha}$ имеет кратность $p_{\varphi} \geqslant 2, \lambda_{u}^{\alpha}$ имеет кратность 1 , а 0 кратность $\mu \geqslant 1$. Остальные элементы этих матриц равны нулю.

Поскольку векторы $n_{1}, \ldots, n_{q+q_{1}}$ являются ненулевыми, то $\lambda_{(\varphi)}^{\alpha} \neq 0, \lambda_{u}^{\beta} \neq 0$ при некоторых значениях индексов $\alpha$ и $\beta$. Более того, так как все эти векторы различны, то

$$
\lambda_{(\varphi)}^{\alpha} \neq \lambda_{(\psi)}^{\alpha} \quad(\varphi \neq \psi), \quad \lambda_{(\varphi)}^{\beta} \neq \lambda_{u}^{\beta}, \quad \lambda_{u}^{\gamma} \neq \lambda_{v}^{\gamma} \quad(u \neq v)
$$

при некоторых значениях индексов $\alpha, \beta, \gamma$.

Обратно, пусть в некотором поле адаптированного ортонормированного репера к $m$-мерному подмногообразию $M$ в евклидовом пространстве $E_{n}$ матрицы $\left\|h_{i j}^{\alpha}\right\|$ второй фундаментальной формы одновременно приводятся к указанному виду, а их диагональные элементы обладают вышеуказанными свойствами и выполняются условия (3.1). Тогда $M$ является нормально плоским минимальным полуэйнштейновым подмногообразием индекса дефектности $\mu \geqslant 1$ с $q$ различными ненулевыми главными векторами кривизны кратностей $p_{1} \geqslant 2$, $\ldots, p_{q} \geqslant 2$ соответственно и $q_{1}$ ненулевыми однократными главными векторами кривизны.

Пусть $T_{x}^{(0)}$ - пространство дефектности подмногообразия $M$ в точке $x$, и пусть $T_{x}^{(1, \varphi)}$ обозначает $p_{\varphi}$-мерное подпространство касательного пространства 
$T_{x}(M)$, на котором каждая матрица $\left\|h_{i j}^{\alpha}\right\|$ имеет собственное значение $\lambda_{(\varphi)}^{\alpha}$ кратности $p_{\varphi}$. Через $T_{x}^{\left(1, \varphi_{1}\right)}$ обозначим одномерное подпространство в $T_{x}(M)$, на котором каждая матрица $\left\|h_{i j}^{\alpha}\right\|$ имеет собственное значение $\lambda_{p+\varphi_{1}-q}^{\alpha}$. Именно в этом смысле будем говорить, что $T_{x}^{(1, \varphi)}$ является собственным подпространством, соответствующим вектору $n_{\varphi}$, а $T_{x}^{\left(1, \varphi_{1}\right)}$ - собственным подпространством, соответствующим вектору $n_{\varphi_{1}}$. Отметим, что по построению подпространства $T_{x}^{(1, \varphi)}$ и $T_{x}^{\left(1, \varphi_{1}\right)}$ имеют максимально возможную размерность, т.е. не допускают расширений, которые обладали бы таким же свойствами, что и $T_{x}^{(1, \varphi)}$ и $T_{x}^{\left(1, \varphi_{1}\right)}$. Справедливо следующее разложение в прямую сумму:

$$
T_{x}(M)=T_{x}^{(1)}+T_{x}^{(0)}=T_{x}^{(1,1)}+\cdots+T_{x}^{(1, q)}+T_{x}^{(1, q+1)}+\cdots+T_{x}^{\left(1, q+q_{1}\right)}+T_{x}^{(0)} .
$$

В дальнейшем будем считать, что адаптированный ортонормированный репер выбран так, что

$$
e_{i_{\varphi}} \in T_{x}^{(1, \varphi)}, \quad e_{p+\varphi_{1}-q} \in T_{x}^{\left(1, \varphi_{1}\right)}, \quad e_{r} \in T_{x}^{(0)} .
$$

Далее, поскольку нормальная связность подмногообразия $M$ плоская, то нормальные к $M$ векторные поля $e_{\alpha}$ можно выбрать так, чтобы они были параллельными в нормальном расслоении, что равносильно условию $\omega_{\beta}^{\alpha}=0$ (см. [23]). В дальнейшем мы будем предполагать, что $e_{\alpha}$ так и выбраны. Подставляя значения $h_{i j}^{\alpha}=\lambda_{i}^{\alpha} \delta_{i j}$ в $(2.1)$ и $(2.2)$, получим следующие равенства:

$$
\begin{gathered}
\omega^{\alpha}=0, \quad \omega_{i}^{\alpha}=\lambda_{i}^{\alpha} \delta_{i j} \omega^{j}, \\
d \lambda_{i}^{\alpha} \delta_{i j}+\left(\lambda_{j}^{\alpha}-\lambda_{i}^{\alpha}\right) \omega_{j}^{i}=h_{i j k}^{\alpha} \omega^{k},
\end{gathered}
$$

где в левой части (3.3) нет суммирования по $i$.

ЛЕмма 1. В выбранном выше репере функиии $h_{i j k}^{\alpha}$ удовлетворяют следующим условиям:

$$
\begin{gathered}
h_{i_{\varphi} j_{\varphi} k}^{\alpha}=0, \quad i_{\varphi} \neq j_{\varphi}, \quad h_{r s k}^{\alpha}=0, \quad h_{i_{\varphi} i_{\varphi} i_{\varphi}}^{\alpha}=0, \quad h_{i_{\varphi} i_{\varphi} j_{\psi}}^{\alpha}=0, \quad \varphi \neq \psi, \\
h_{i_{\varphi} i_{\varphi} u}^{\alpha}=h_{j_{\varphi} j_{\varphi} u}^{\alpha}, \quad h_{i_{\varphi} i_{\varphi} r}^{\alpha}=h_{j_{\varphi} j_{\varphi} r}^{\alpha}, \quad i_{\varphi} \neq j_{\varphi}, \\
\sum_{\alpha} \lambda_{(\varphi)}^{\alpha} h_{i_{\varphi} i_{\varphi} u}^{\alpha}=\sum_{\alpha} \lambda_{(\chi)}^{\alpha} h_{j_{\chi} j_{\chi} u}^{\alpha}, \quad \sum_{\alpha} \lambda_{(\varphi)}^{\alpha} h_{i_{\varphi} i_{\varphi} r}^{\alpha}=\sum_{\alpha} \lambda_{(\chi)}^{\alpha} h_{j_{\chi} j_{\chi} r}^{\alpha}, \quad \varphi \neq \chi .
\end{gathered}
$$

ДокАЗАТЕЛЬСтво. Пусть в (3.3)

$$
i=i_{\varphi}, \quad j=j_{\varphi}, \quad i_{\varphi} \neq j_{\varphi}
$$

Тогда левая часть равна нулю, и в силу независимости форм $\omega^{k}$ имеем

$$
h_{i_{\varphi} j_{\varphi} k}^{\alpha}=0 .
$$

Если в (3.3) $i=r, j=s$, то $h_{r s k}^{\alpha}=0$ в силу того, что $\lambda_{r}^{\alpha}=\lambda_{s}^{\alpha}=0$. Пусть в $(3.3)$ $i=i_{\varphi}, j=i_{\varphi}$. Тогда с учетом полученных равенств будем иметь

$$
d \lambda_{(\varphi)}^{\alpha}=h_{i_{\varphi} i_{\varphi} i_{\varphi}}^{\alpha} \omega^{i_{\varphi}}+\sum_{\substack{\psi \\(\psi \neq \varphi)}} h_{i_{\varphi} i_{\varphi} j_{\psi}}^{\alpha} \omega^{j_{\psi}}+h_{i_{\varphi} i_{\varphi} u}^{\alpha} \omega^{u}+h_{i_{\varphi} i_{\varphi} r}^{\alpha} \omega^{r}
$$


где в первом слагаемом правой части нет суммирования по индексу $i_{\varphi}$. Если в (3.3) $i=j_{\varphi}, j=j_{\varphi}, j_{\varphi} \neq i_{\varphi}$, то точно так же получим

$$
d \lambda_{(\varphi)}^{\alpha}=h_{j_{\varphi} j_{\varphi} j_{\varphi}}^{\alpha} \omega^{j_{\varphi}}+\sum_{\substack{\psi \\(\psi \neq \varphi)}} h_{j_{\varphi} j_{\varphi} j_{\psi}}^{\alpha} \omega^{j_{\psi}}+h_{j_{\varphi} j_{\varphi} u}^{\alpha} \omega^{u}+h_{j_{\varphi} j_{\varphi} r}^{\alpha} \omega^{r}
$$

где в первом слагаемом правой части нет суммирования по индексу $j_{\varphi}$. Сравнивая полученные равенства, приходим к следующим соотношениям:

$$
\begin{gathered}
h_{i_{\varphi} i_{\varphi} i_{\varphi}}^{\alpha}=0, \quad h_{i_{\varphi} i_{\varphi} u}^{\alpha}=h_{j_{\varphi} j_{\varphi} u}^{\alpha}, \quad h_{i_{\varphi} i_{\varphi} r}^{\alpha}=h_{j_{\varphi} j_{\varphi} r}^{\alpha}, \quad i_{\varphi} \neq j_{\varphi}, \\
h_{i_{\varphi} i_{\varphi} j_{\psi}}^{\alpha}=h_{j_{\varphi} j_{\varphi} j_{\psi}}^{\alpha}, \quad i_{\varphi} \neq j_{\varphi}, \quad \psi \neq \varphi \\
d \lambda_{(\varphi)}^{\alpha}=\sum_{\substack{\psi \\
(\psi \neq \varphi)}} h_{i_{\varphi} i_{\varphi} j_{\psi}}^{\alpha} \omega^{j_{\psi}}+h_{i_{\varphi} i_{\varphi} u}^{\alpha} \omega^{u}+h_{i_{\varphi} i_{\varphi} r}^{\alpha} \omega^{r} .
\end{gathered}
$$

Умножая равенство (3.4) на $\lambda_{(\varphi)}^{\alpha}$ и суммируя по $\alpha$, получим (см. (3.1))

$$
\frac{1}{2} d \rho=\sum_{\substack{\psi \\(\neq \neq \varphi)}}\left(\sum_{\alpha} \lambda_{(\varphi)}^{\alpha} h_{i_{\varphi} i_{\varphi} j_{\psi}}^{\alpha}\right) \omega^{j \psi}+\sum_{\alpha} \lambda_{(\varphi)}^{\alpha} h_{i_{\varphi} i_{\varphi} u}^{\alpha} \omega^{u}+\sum_{\alpha} \lambda_{(\varphi)}^{\alpha} h_{i_{\varphi} i_{\varphi} r}^{\alpha} \omega^{r} .
$$

Из этого равенства следует, что фактически его правая часть не зависит ни от субиндекса $\varphi$, ни от индекса $i_{\varphi}$, т.е.

$$
\frac{1}{2} d \rho=\sum_{\substack{\psi \\(\psi \neq \chi)}}\left(\sum_{\alpha} \lambda_{(\chi)}^{\alpha} h_{j_{\chi} j_{\chi} j_{\psi}}^{\alpha}\right) \omega^{j_{\psi}}+\sum_{\alpha} \lambda_{(\chi)}^{\alpha} h_{j_{\chi} j_{\chi} u}^{\alpha} \omega^{u}+\sum_{\alpha} \lambda_{(\chi)}^{\alpha} h_{j_{\chi} j_{\chi} r}^{\alpha} \omega^{r}
$$

где $\chi \neq \varphi$. Сравнивая это равенство с (3.5), приходим к следующим соотношениям:

$$
\begin{aligned}
\sum_{\alpha} \lambda_{(\varphi)}^{\alpha} h_{i_{\varphi} i_{\varphi} u}^{\alpha}= & \sum_{\alpha} \lambda_{(\chi)}^{\alpha} h_{j_{\chi} j_{\chi} u}^{\alpha}, \quad \sum_{\alpha} \lambda_{(\varphi)}^{\alpha} h_{i_{\varphi} i_{\varphi} r}^{\alpha}=\sum_{\alpha} \lambda_{(\chi)}^{\alpha} h_{j_{\chi} j_{\chi} r}^{\alpha}, \\
& \sum_{\alpha} \lambda_{(\varphi)}^{\alpha} h_{i_{\varphi} i_{\varphi} j_{\psi}}^{\alpha}=0, \quad \psi \neq \varphi .
\end{aligned}
$$

Если в (3.3) положить $i=i_{\varphi}, j=j_{\psi}, \varphi \neq \psi$, то будем иметь

$$
\left(\lambda_{(\psi)}^{\alpha}-\lambda_{(\varphi)}^{\alpha}\right) \omega_{j_{\psi}}^{i_{\varphi}}=h_{i_{\varphi} i_{\varphi} j_{\psi}}^{\alpha} \omega^{i_{\varphi}}+h_{i_{\varphi} j_{\psi} k}^{\alpha} \omega^{k},
$$

где в правой части нет суммирования по индексу $i_{\varphi}$, а индекс $k$ не принимает значений $j_{\varphi}$. Умножая это равенство на $\lambda_{(\varphi)}^{\alpha}$ и суммируя по $\alpha$, а также учитывая $(*)$, получим

$$
\rho\left(\cos \theta_{\varphi \psi}-1\right) \omega_{j_{\psi}}^{i_{\varphi}}=\sum_{\alpha} \lambda_{(\varphi)}^{\alpha} h_{i_{\varphi} j_{\psi} k}^{\alpha} \omega^{k}
$$

где $k \neq j_{\varphi}$. Поскольку $n_{\varphi} \neq n_{\psi}$, то выражение в круглых скобках отлично от нуля, и мы приходим к выводу, что форма $\omega_{j_{\psi}}^{i_{\varphi}}$ не зависит от $\omega^{i_{\varphi}}$. Аналогично доказывается, что $\omega_{j_{\psi}}^{i_{\varphi}}$ не зависит также от $\omega^{j_{\psi}}$. Отсюда следует, что $h_{i_{\varphi} i_{\varphi} j_{\psi}}^{\alpha}=0$ при $\varphi \neq \psi$. Лемма доказана. 
Лемма 2. Справедлива следующая система соотношений:

$$
\begin{gathered}
\left(\lambda_{(\psi)}^{\alpha}-\lambda_{(\varphi)}^{\alpha}\right) \omega_{j_{\psi}}^{i_{\varphi}}=\sum_{\substack{\chi \\
(\chi \neq \varphi, \chi \neq \psi)}} h_{i_{\varphi} j_{\psi} k_{\chi}}^{\alpha} \omega^{k_{\chi}}+h_{i_{\varphi} j_{\psi} u}^{\alpha} \omega^{u}+h_{i_{\varphi} j_{\psi} r}^{\alpha} \omega^{r}, \quad \varphi \neq \psi \\
\left(\lambda_{u}^{\alpha}-\lambda_{v}^{\alpha}\right) \omega_{u}^{v}=h_{u v k}^{\alpha} \omega^{k} \\
\left(\lambda_{(\varphi)}^{\alpha}-\lambda_{u}^{\alpha}\right) \omega_{j_{\varphi}}^{u}=h_{u j_{\varphi} k}^{\alpha} \omega^{k} \\
\lambda_{u}^{\alpha} \omega_{u}^{r}=h_{u r a}^{\alpha} \omega^{a}+h_{u r v}^{\alpha} \omega^{v} \\
\lambda_{(\varphi)}^{\alpha} \omega_{j_{\varphi}}^{r}=h_{r j_{\varphi} a}^{\alpha} \omega^{a}+h_{r j_{\varphi} u}^{\alpha} \omega^{u} \\
d \lambda_{(\varphi)}^{\alpha}=h_{i_{\varphi} i_{\varphi} u}^{\alpha} \omega^{u}+h_{i_{\varphi} i_{\varphi} r}^{\alpha} \omega^{r} \\
d \lambda_{u}^{\alpha}=h_{u u k}^{\alpha} \omega^{k}
\end{gathered}
$$

где в левых частях (3.8) и (3.9) нет суммирования по индексам $v$ и и соответственно.

ДоказАТельство. Равенства (3.7) и (3.12) следуют соответсвенно из (3.6) и (3.4) с учетом того, что $h_{i_{\varphi} i_{\varphi} j_{\psi}}^{\alpha}=0$ при $\varphi \neq \psi$. Равенства (3.8)-(3.11) и (3.13) получаются из (3.3) при соответствующих значениях индексов $i$ и $j$. Например, если в (3.3) $i=r, j=u$, то получаем (3.10), где учтено, что $h_{r s k}^{\alpha}=0$. Лемма доказана.

Умножая (3.11) на $\lambda_{(\varphi)}^{\alpha}$ и суммируя по $\alpha$, получим

$$
\omega_{j_{\varphi}}^{r}=F_{r} \omega^{j_{\varphi}}+\frac{1}{\rho} \sum_{\substack{\psi \\(\psi \neq \varphi)}}\left(\sum_{\alpha} \lambda_{(\varphi)}^{\alpha} h_{r j_{\varphi} j_{\psi}}^{\alpha}\right) \omega^{j_{\psi}}+\frac{1}{\rho}\left(\sum_{\alpha} \lambda_{(\varphi)}^{\alpha} h_{r j_{\varphi} u}^{\alpha}\right) \omega^{u},
$$

где

$$
F_{r}=\frac{1}{\rho} \sum_{\alpha} \lambda_{(\varphi)}^{\alpha} h_{j_{\varphi} j_{\varphi} r}^{\alpha} .
$$

Из последнего равенства в формулировке леммы 1 следует, что функции $F_{r}$ не зависят ни от значения субиндекса $\varphi$, ни от значения индекса $j_{\varphi}$.

Далее, если умножить $(3.12)$ на $\lambda_{(\varphi)}^{\alpha}$ и просуммировать по $\alpha$, а затем разделить на $\rho$, то получим

$$
\frac{1}{2} d \ln \rho=G_{u} \omega^{u}+F_{r} \omega^{r}
$$

где

$$
G_{u}=\frac{1}{\rho} \sum_{\alpha} \lambda_{(\varphi)}^{\alpha} h_{i_{\varphi} i_{\varphi} u}^{\alpha}
$$

Из предпоследнего равенства в формулировке леммы 1 следует, что функции $G_{u}$ также не зависят ни от значения субиндекса $\varphi$, ни от значения индекса $i_{\varphi}$.

Если (3.9) умножить на $\lambda_{(\varphi)}^{\alpha}$ и просуммировать по $\alpha$, а затем разделить на $\rho$, то получим следующее равенство:

$$
\begin{array}{r}
\left(1-\cos \theta_{\varphi \psi_{u}}\right) \omega_{j_{\varphi}}^{u}=G_{u} \omega^{j_{\varphi}}+\frac{1}{\rho} \sum_{\substack{\psi \\
(\psi \neq \varphi)}}\left(\sum_{\alpha} \lambda_{(\varphi)}^{\alpha} h_{u j_{\varphi} j_{\psi}}^{\alpha}\right) \omega^{j_{\psi}} \\
+\frac{1}{\rho}\left(\sum_{\alpha} \lambda_{(\varphi)}^{\alpha} h_{u j_{\varphi} v}^{\alpha}\right) \omega^{v}+\frac{1}{\rho}\left(\sum_{\alpha} \lambda_{(\varphi)}^{\alpha} h_{u j_{\varphi} r}^{\alpha}\right) \omega^{r},
\end{array}
$$

где $\psi_{u}=u-p+q, \theta_{\varphi \psi_{u}}=n_{\varphi} \wedge n_{u-p+q}$ и в левой части нет суммирования по $u$. 
Лемма 3. Распределение $T^{(1, \varphi)}$ является интегрируемым, а его интегральное многообразие представляет собой $p_{\varphi}$-мерную сферу $S^{p_{\varphi}}\left(R_{\varphi}\right)$. Если подмногообразие $M$ не имеет однократных главных векторов кривизны, то все сферы имеют один и тот же радиус $R$.

ДокАЗАТЕльство. Распределение $T^{(1, \varphi)}$ задается следующей пфаффовой системой:

$$
\omega^{\alpha}=0, \quad \omega^{i_{\psi}}=0, \quad \psi \neq \varphi, \quad \omega^{u}=0, \quad \omega^{r}=0 .
$$

При выполнении этих условий (3.7), (3.14), (3.16) принимают соответственно следующий вид:

$$
\omega_{i_{\varphi}}^{j \psi}=0, \quad \omega_{j_{\varphi}}^{r}=F_{r} \omega^{j_{\varphi}}, \quad \omega_{j_{\varphi}}^{u}=\frac{G_{u}}{1-\cos \theta_{\varphi \psi_{u}}} \omega^{j_{\varphi}},
$$

где первое равенство получено из (3.7) на основании того, что $\lambda_{(\varphi)}^{\alpha} \neq \lambda_{(\psi)}^{\alpha}$ хотя бы при одном значении индекса $\alpha$. Так как, кроме того, $\omega_{j_{\varphi}}^{\alpha}=\lambda_{(\varphi)}^{\alpha} \omega^{j_{\varphi}}$, то непосредственно проверяется, что

$$
d \omega^{\alpha}=0, \quad d \omega^{i} \psi=0, \quad d \omega^{u}=0, \quad d \omega^{r}=0 .
$$

Отсюда следует, что система (3.17) вполне интегрируема. Из выражений для $\omega_{i_{\varphi}}^{j_{\psi}}, \omega_{j_{\varphi}}^{r}, \omega_{j_{\varphi}}^{u}, \omega_{j_{\varphi}}^{\alpha}$ следует, что интегральное многообразие системы (3.17) является вполне омбилическим в $E_{n}$ (но не вполне геодезическим, так как $\lambda_{(\varphi)}^{\alpha} \neq 0$ при некотором значении индекса $\alpha$ ), т.е. локально представляет собой $p_{\varphi}$-мерную сферу $S^{p_{\varphi}}\left(R_{\varphi}\right)$. Поскольку вектор средней кривизны $H_{\varphi}$ этой сферы определяется равенством

$$
H_{\varphi}=\lambda_{(\varphi)}^{\alpha} e_{\alpha}+G_{\varphi}^{u} e_{u}+\sum_{r} F_{r} e_{r}
$$

где

$$
G_{\varphi}^{u}=G_{u} \cdot\left(1-\cos \theta_{\varphi \psi_{u}}\right)^{-1},
$$

то для радиуса $R_{\varphi}$ получаем следующую формулу:

$$
R_{\varphi}^{2}=\frac{1}{\left|H_{\varphi}\right|^{2}}=\left(\rho+\sum_{u}\left(G_{\varphi}^{u}\right)^{2}+\sum_{r} F_{r}^{2}\right)^{-1}
$$

Отсюда следует, что значение радиуса $R_{\varphi}$ зависит от $\varphi$. Если подмногообразие $M$ не имеет однократных главных векторов кривизны, то множество значений индекса $u$ является пустым, и последнее равенство будет иметь следующий вид:

$$
R_{\varphi}^{2}=\left(\rho+\sum_{r} F_{r}^{2}\right)^{-1} .
$$

Поскольку правая часть не зависит от $\varphi$, то $R_{1}=R_{2}=\cdots=R_{q}=R$, т.е. все сферы имеют один и тот же радиус. Лемма доказана.

Лемма 4. Распределение $T^{(0)}$ является интегрируемым, и его интегральное многообразие $M^{(0)}$ представляет собой н-мерную плоскость. 
ДокАЗАТЕЛЬство. Распределение $T^{(0)}$ задается пфаффовой системой

$$
\omega^{\alpha}=0, \quad \omega^{a}=0, \quad \omega^{u}=0 .
$$

Из (3.10) и (3.14) следует, что в этом случае $\omega_{r}^{u}=0$ (так как $\lambda_{u}^{\alpha} \neq 0$ при некотором значении индекса $\alpha)$ и $\omega_{a}^{r}=0$. Поскольку, кроме того, $\omega_{r}^{\alpha}=0$, то легко проверяется, что $d \omega^{\alpha}=0, d \omega^{a}=0, d \omega^{u}=0$, и система (3.18) вполне интегрируема. Из этих же равенств следует, что вторая фундаментальная форма интегрального многообразия $M^{(0)}$ распределения $T^{(0)}$ нулевая. Это значит, что $M^{(0)}$ является вполне геодезическим подмногообразием в $E_{n}$, т.е. представляет собой $\mu$-мерную плоскость. Лемма доказана.

Лемма 5. На подмногообразии М распределение

$$
\widetilde{T}^{(1)}=T^{(1,1)}+\cdots+T^{(1, q)},
$$

сопоставляющее каждой точке $x \in M$ прямую сумму

$$
\widetilde{T}_{x}^{(1)}=T_{x}^{(1,1)}+\cdots+T_{x}^{(1, q)},
$$

является интегрируемым тогда и только тогда, когда распределения $T^{(1,1)}$, $\ldots, T^{(1, q)}$ параллельны друг относительно друга в направлении подпространства

$$
P_{x}=T_{x}^{(1, q+1)}+\cdots+T_{x}^{\left(1, q+q_{1}\right)}+T_{x}^{(0)} .
$$

Если $q=2$ и $\widetilde{T}^{(1)}$ интегрируемо, то распределения $T^{(1,1)}$ и $T^{(1,2)}$ параллельны на всем $M$.

ДокАЗАтЕльство. Распределение $\widetilde{T}^{(1)}$ задается пфаффовой системой

$$
\omega^{\alpha}=0, \quad \omega^{u}=0, \quad \omega^{r}=0 .
$$

Предположим, что эта система вполне интегрируема, и через $M^{(1)}$ обозначим ее интегральное многообразие. Поскольку $\omega_{r}^{\alpha}=0$ и $\omega_{u}^{\alpha}=\lambda_{u}^{\alpha} \omega^{u}$ (нет суммирования), то в силу (3.19) $\omega_{u}^{\alpha}=0$ и, следовательно, $T^{\perp}(M)$ и $T^{(1, q+1)}+$ $\cdots+T^{\left(1, q+q_{1}\right)}+T^{(0)}$ как подрасслоения нормального расслоения подмногообразия $M^{(1)}$ параллельны в нормальном расслоении; кроме того, связность в $T^{\perp}(M)$, как мы знаем, плоская. Так как векторные поля $e_{\alpha}$ в силу их выбора являются параллельными в нормальном расслоении на $M$, то они будут параллельными и в нормальном расслоении на $M^{(1)}$. Тогда они будут коммутируемыми в смысле работы [29]. Это значит, что матрицы $\left\|\bar{h}_{a b}^{\alpha}\right\|$ второй фундаментальной формы подмногообразия $M^{(1)}$, соответствующие векторам $e_{\alpha}$, коммутируют со всеми остальными матрицами второй фундаментальной формы этого подмногообразия и, в частности, со всеми матрицами $\left\|\bar{h}_{a b}^{u}\right\|$ и $\left\|\bar{h}_{a b}^{r}\right\|$, которые соответствуют нормальным к $M^{(1)}$ векторам $e_{u}$ и $e_{r}$ соответственно. Из (3.11) следует, что если $\lambda_{(\varphi)}^{\alpha}=0$, то $h_{r j_{\varphi} j_{\psi}}^{\alpha}=0$ при $\psi \neq \varphi$. Если же $\lambda_{(\varphi)}^{\alpha} \neq 0$, то из (3.11) получаем

$$
\omega_{j_{\varphi}}^{r}=F_{r} \omega^{j_{\varphi}}+\sum_{\substack{\psi \\(\psi \neq \varphi)}}\left(\lambda_{(\varphi)}^{\alpha}\right)^{-1} h_{r j_{\varphi} j_{\psi}}^{\alpha} \omega^{j_{\psi}},
$$


где правая часть фактически не зависит от $\alpha$. Из этого равенства следует, что каждая матрица $\left\|\bar{h}_{a b}^{r}\right\|$ имеет следующий вид:

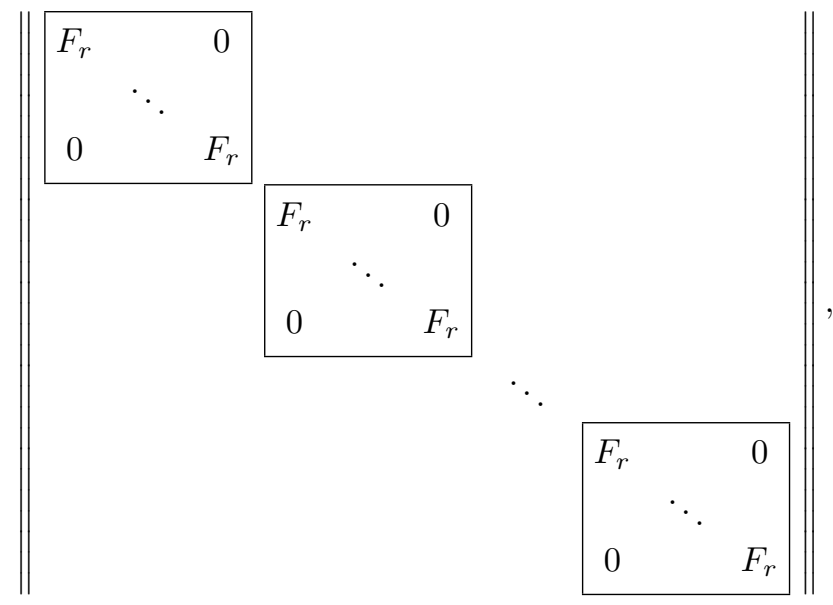

где на остальных местах стоят элементы вида $\left(\lambda_{(\varphi)}^{\alpha}\right)^{-1} h_{r j_{\varphi} j_{\psi}}^{\alpha}$. Поскольку $\omega_{a}^{\alpha}=$ $\lambda_{a}^{\alpha} \omega^{a}$ (нет суммирования), то матрицы $\left\|\bar{h}_{a b}^{\alpha}\right\|$ имеют диагональный вид с диагональными элементами $\lambda_{a}^{\alpha}$ соответствующей кратности. Подпространства $T_{x}^{(1, \varphi)}$ являются инвариантными подпространствами этих матриц, причем каждая матрица $\left\|\bar{h}_{a b}^{\alpha}\right\|$ имеет на $T_{x}^{(1, \varphi)}$ только одно собственное значение $\lambda_{(\varphi)}^{\alpha}$. Так как матрицы $\left\|\bar{h}_{a b}^{r}\right\|$ коммутируют со всеми матрицами $\left\|\bar{h}_{a b}^{\alpha}\right\|$, то подпространства $T_{x}^{(1, \varphi)}$ будут инвариантными также и для матриц $\left\|\bar{h}_{a b}^{r}\right\|$. Отсюда следует, что в выбранном репере матрицы $\left\|\bar{h}_{a b}^{r}\right\|$ должны иметь блочно-диагональный вид. Следовательно, в матрице (3.20) указанные диагональные блоки остаются, а все остальные элементы равны нулю, т.е. $h_{r j_{\varphi} j_{\psi}}^{\alpha}=0$ при $\varphi \neq \psi$. Так как $\lambda_{(\varphi)}^{\alpha} \neq \lambda_{u}^{\alpha}$ при некотором значении индекса $\alpha$, то из (3.9) (с учетом условий (3.19)) получаем

$$
\omega_{j_{\varphi}}^{u}=\widetilde{G}_{\varphi}^{u} \omega^{j_{\varphi}}+\left(\lambda_{(\varphi)}^{\alpha}-\lambda_{u}^{\alpha}\right)^{-1} \sum_{\substack{\psi \\(\psi \neq \varphi)}} h_{u j_{\varphi} j_{\psi}}^{\alpha} \omega^{j_{\varphi}},
$$

где $\widetilde{G}_{\varphi}^{u}=\left(\lambda_{(\varphi)}^{\alpha}-\lambda_{u}^{\alpha}\right)^{-1} h_{u j_{\varphi} j_{\psi}}^{\alpha}$. Отсюда следует, что матрицы $\left\|\bar{h}_{a b}^{u}\right\|$ имеют такой же вид, как и (3.20), но с диагональными элементами $\widetilde{G}_{\varphi}^{u}$ в соответствующих блоках. На остальных местах стоят элементы вида $\left(\lambda_{(\varphi)}^{\alpha}-\lambda_{u}^{\alpha}\right)^{-1} h_{u j_{\varphi} j_{\psi}}^{\alpha}$. Поскольку матрицы $\left\|\bar{h}_{a b}^{u}\right\|$ коммутируют с матрицами $\left\|\bar{h}_{a b}^{\alpha}\right\|$, то, рассуждая, как и выше, получим, что $h_{u j_{\varphi} j_{\psi}}^{\alpha}=0$ при $\varphi \neq \psi$. Тогда из (3.7) следует, что

$$
\left(\lambda_{(\psi)}^{\alpha}-\lambda_{\varphi}^{\alpha}\right) \omega_{j_{\psi}}^{i_{\varphi}}=\sum_{\substack{\chi \\(\chi \neq \varphi, \chi \neq \psi)}} h_{i_{\varphi} j_{\psi} k_{\chi}}^{\alpha} \omega^{k_{\chi}}, \quad \varphi \neq \psi
$$

Поскольку $\lambda_{\varphi}^{\alpha} \neq \lambda_{(\psi)}^{\alpha}$ при некотором значении $\alpha$, то из этого равенства получаем, что $\omega_{j_{\psi}}^{i_{\varphi}}=0$ при $\omega^{k_{\chi}}=0$. Это и значит, что распределения $T^{(1, \varphi)}, T^{(1, \psi)}$ при $\varphi \neq \psi$ параллельны друг относительно друга в направлении подпространства $P_{x}$. Если $q=2$, то множество значений индекса $k_{\chi}$ пусто и, следовательно, 
$\omega_{j_{2}}^{i_{1}}=0$. Это и значит, что распределения $T^{(1,1)}, T^{(1,2)}$ параллельны друг относительно друга на всем подмногообразии $M$. Обратно, если $\omega_{j_{\psi}}^{i_{\varphi}}=0$ при $\omega^{k_{\chi}}=0, \varphi \neq \psi$, то из (3.7) следует, что $h_{u i_{\varphi} j_{\psi}}^{\alpha}=0, h_{r i_{\varphi} j_{\psi}}^{\alpha}=0$ при $\varphi \neq \psi$. Если $q=2$, то при $\omega_{j_{2}}^{i_{1}}=0$ имеем $h_{u i_{1} j_{2}}^{\alpha}=0, h_{r i_{1} j_{2}}^{\alpha}=0$. Покажем, что при выполнении этих условий система (3.19) вполне интегрируема. Действительно, поскольку $\omega_{a}^{\alpha}=\lambda_{a}^{\alpha} \omega^{a}$ (нет суммирования), то легко проверяется, что $d \omega^{\alpha}=0$. Далее, в силу условий (3.19) и условия $h_{u i_{\varphi} j_{\psi}}^{\alpha}=0, \varphi \neq \psi$, из (3.16) следует, что $\omega_{j_{\varphi}}^{u}=G_{\varphi}^{u} \omega^{j \varphi}$. Следовательно,

$$
d \omega^{u}=\sum_{\varphi} \omega^{j_{\varphi}} \wedge \omega_{j_{\varphi}}^{u}=0
$$

Точно так же с использованием условий (3.19), (3.14) и условия $h_{r i_{\varphi} j_{\psi}}^{\alpha}=0$, $\varphi \neq \psi$, легко показать, что $d \omega^{r}=0$. Следовательно, система (3.19) вполне интегрируема. Лемма доказана.

В ходе доказательства леммы 5 фактически было доказано, что в выбранном на подмногообразие $M$ репере равенства $h_{u i_{\varphi} j_{\psi}}^{\alpha}=0, h_{r i_{\varphi} j_{\psi}}^{\alpha}=0$ при $\varphi \neq \psi$ являются необходимыми и достаточными условиями как интегрируемости распределения $\widetilde{T}^{(1)}$, так и параллельности распределений $T^{(1,1)}, \ldots, T^{(1, q)}$ друг относительно друга в направлении подпространства $P_{x}$.

ЛЕмма 6. Пусть распределение $\widetilde{T}^{(1)}$, определенное на подмногообразии $M$, как в лемме 5, является интегрируемым. Тогда его интегралъное многообразие $M^{(1)}$ имеет плоскую нормальную связность. Если подмногообразие $M$ не имеет однократных главных векторов кривизны, то $M^{(1)}$ является эйнштейновым подмногообразием в $E_{n}$.

ДокАЗАТЕЛЬСтво. Поскольку распределение $\widetilde{T}^{(1)}$ задается системой (3.19) и, кроме того, $h_{u i_{\varphi} j_{\psi}}^{\alpha}=0, h_{r i_{\varphi} j_{\psi}}^{\alpha}=0$ при $\varphi \neq \psi$, то $\omega_{j_{\varphi}}^{r}=F_{r} \omega^{j_{\varphi}}, \omega_{j_{\varphi}}^{u}=G_{\varphi}^{u} \omega^{j_{\varphi}}$ и, как мы знаем, $\omega_{j_{\varphi}}^{\alpha}=\lambda_{(\varphi)}^{\alpha} \omega^{j_{\varphi}}$. Следовательно, все матрицы второй фундаментальной формы подмногообразия $M^{(1)}$ одновременно приведены к диагональному виду. Согласно теореме Картана (см. [23]) подмногообразие $M^{(1)}$ имеет плоскую нормальную связность. Тензор Риччи подмногообразия $M^{(1)}$ также имеет диагональный вид (см. §2) с диагональными элементами $\widetilde{\rho}_{a}$, которые можно вычислить следующим образом. Пусть

$$
\begin{gathered}
\tilde{n}_{a}=\lambda_{a}^{\alpha} e_{\alpha}+G_{a}^{u} e_{u}+F^{r} e_{r} \\
\widetilde{H}=\left(\sum_{b} \lambda_{b}^{\alpha}\right) e_{\alpha}+\left(\sum_{c} G_{c}^{u}\right) e_{u}+\left(p_{1}+\cdots+p_{q}\right) F^{r} e_{r},
\end{gathered}
$$

(где $F^{r}=F_{r}$ ), обозначают соответственно главные векторы кривизны и вектор средней кривизны подмногообразия $M^{(1)}$, где $G_{a}^{u}=G_{\varphi}^{u}$ при $a=i_{\varphi}$ (функции $G_{\varphi}^{u}$ определены при доказательстве леммы 3$)$. Тогда, проводя вычисления по общей формуле (2.3), получим

$$
\widetilde{\rho}_{a}=\sum_{\alpha}\left\{\left(\lambda_{a}^{\alpha}\right)^{2}-\sum_{b} \lambda_{a}^{\alpha} \lambda_{b}^{\alpha}\right\}+\sum_{u}\left\{\left(G_{a}^{u}\right)^{2}-\sum_{c} G_{a}^{u} G_{c}^{u}\right\}-\left(p_{1}+\cdots+p_{q}-1\right) \sum_{r}\left(F^{r}\right)^{2} .
$$


Так как в этой формуле правая часть существенно зависит от индекса $a$, то подмногообразие $M^{(1)}$, вообще говоря, не является эйнштейновым. Если подмногообразие $M$ не имеет однократных главных векторов кривизны, то $\lambda_{u}^{\alpha}=0$, $\sum_{a} \lambda_{a}^{\alpha}=0($ см. $(3.1))$ и $G_{a}^{u}=0$. Тогда

$$
\tilde{\rho}_{a}=\rho-\left(p_{1}+\cdots+p_{q}-1\right) \sum_{r}\left(F^{r}\right)^{2}
$$

для любого значения $a$, и подмногообразие $M^{(1)}$ является эйнштейновым. Лемма доказана.

ЗАмечАниЕ 1 . Отметим, что в формуле (3.23) случай $\widetilde{\rho}_{a}=0$ не исключается. В этом случае $M^{(1)}$ является риччи-плоским. Если в $(3.23) F^{r}=0$, то $M^{(1)}$ - эйнштейново подмногообразие с ненулевой эйнштейновой константой $\rho$.

ЛЕмма 7. Если на подмногообразии М распределение $\widetilde{T}^{(1)}$ интегрируемо и $q \geqslant 3$, то его интегральное многообразие $M^{(1)}$ является либо прямым произведением сфер $S^{p_{1}}\left(R_{1}\right), \ldots, S^{p_{q}}\left(R_{q}\right)$, либо скрученным произведением этих сфер, либо прямым произведением вида $N_{0} \times N_{1} \times \cdots \times N_{\tau}$, где $N_{0}$ есть прямое произведение некоторых из этих сфер, а $N_{1}, \ldots, N_{\tau}$ представляют собой скрученные произведения различных групп остальных сфер. При $q=2 M^{(1)}$ всегда является прямым произведением сфер $S^{p_{1}}\left(R_{1}\right), S^{p_{2}}\left(R_{2}\right)$.

ДокАЗАТЕльство. Если $\omega_{j_{\psi}}^{i_{\varphi}}=0$ при $\varphi \neq \psi$, то распределения $T^{(1, \varphi)}$ параллельны на $M^{(1)}$. Поскольку все матрицы второй фундаментальной формы подмногообразия $M^{(1)}$ имеют диагональный вид (лемма 6$)$, то распределения $T^{(1, \varphi)}$ сопряжены относительно этой формы. Следовательно (см. [30]), $M^{(1)}$ является прямым произведением их интегральных многообразий, т.е. сфер $S^{p_{\varphi}}\left(R_{\varphi}\right)$. Далее, из (3.21) следует, что если $q \geqslant 3$, то, вообще говоря, $\omega_{j_{\psi}}^{i_{\varphi}} \neq 0$ при $\varphi \neq \psi$. Геометрически это означает, что подпространства $T_{x}^{(1, \varphi)}$, а следовательно, и сферы $S^{p_{\varphi}}\left(R_{\varphi}\right)$ вращаются друг относительно друга при движении вдоль подмногообразия $M^{(1)}$. В этом случае говорят, что $M^{(1)}$ является скрученным произведением сфер $S^{p_{\varphi}}\left(R_{\varphi}\right)$. Если из распределений $T^{(1, \varphi)}$ можно составить попарно непересекающиеся суммы, которые параллельны на $M^{(1)}$ друг относительно друга, то $M^{(1)}$ будет иметь вид прямого произведения, указанного в формулировке леммы. Последнее утверждение леммы следует из того, что при $q=2$ распределения $T^{(1,1)}$ и $T^{(1,2)}$ всегда параллельны на $M^{(1)}$ (лемма 5$)$. Лемма доказана.

ЗАмечАниЕ 2. Поскольку две сферы не могут образовать скрученного произведения, то, говоря о скрученном произведении сфер, мы будем предполагать, что оно образовано не менее чем тремя сферами.

ЗАмечАнИЕ 3. В [28] рассматривался частный случай теоремы 1 , когда распределение $\widetilde{T}^{(1)}$ совпадает с распределением кодефектности $T^{(1)}$, а распределения $T^{(1, \varphi)}$ параллельны друг относительно друга, но не относительно $T^{(0)}$. Было доказано, что в этом случае главные векторы кривизны $n_{1}, \ldots, n_{q}$ образуют между собой попарно равные углы и, как следствие, имеют равные кратности, т.е. $p_{1}=\cdots=p_{q}$ (см. $\left.\S 2\right)$. 
На основании доказанных выше лемм приходим к следующему важному заключению: если в евклидовом пространстве $E_{n}$ нормально плоское минимальное полуэйнштейново подмногообразие $M$ индекса дефектности $\mu \geqslant 1$ имеет $q \geqslant 2$ различных ненулевых главных векторов кривизны кратностей $p_{1} \geqslant 2$, $\ldots, p_{q} \geqslant 2$ и распределение $\widetilde{T}^{(1)}$, определенное в лемме 5 , интегрируемо, то $M$ локально допускает $\left(p_{1}+\cdots+p_{q}\right)$-мерное слоение, слои которого описываются леммой 7 . Если $M$ не имеет ненулевых однократных главных векторов кривизны, то распределение $\widetilde{T}^{(1)}$ совпадает с распределением кодефектности $T^{(1)}$. Отметим, что в последнем случае равенство $q=1$ невозможно (так как подмногообразие $M$ не будет минимальным), и потому мы рассматриваем случай $q \geqslant 2$.

\section{§ 4. Доказательство теоремы 1}

Ряд утверждений теоремы 1 доказан фактически в леммах 3-7. Здесь мы докажем остальные утверждения теоремы и при одном дополнительном условии (см. (4.3)) обсудим вопрос существования такого подмногообразия.

Пусть подмногообразие $M$ не имеет ненулевых однократных главных векторов кривизны, т.е. $q_{1}=0$. В этом случае распределение $\widetilde{T}^{(1)}$, определенное в лемме 5 , совпадает с распределением кодефектности $T^{(1)}$, а его интегральное многообразие $M^{(1)}$ фактически является интегральным многообразием для $T^{(1)}$.

Поскольку при $q_{1}=0$ множество значений индексов $u, v, \ldots, \varphi_{1}, \psi_{1}, \ldots$ пусто и $h_{r j_{\varphi} j_{\psi}}^{\alpha}=0$ при $\varphi \neq \psi$, то из $(3.12)$, (3.14) и (3.7) получаем соответственно следующие равенства:

$$
\begin{gathered}
d \ln \left|\lambda_{(\varphi)}^{\alpha}\right|=F_{r} \omega^{r}, \\
\omega_{j_{\varphi}}^{r}=F_{r} \omega^{j_{\varphi}}, \\
\left(\lambda_{(\psi)}^{\alpha}-\lambda_{(\varphi)}^{\alpha}\right) \omega_{j_{\psi}}^{i_{\varphi}}=\sum_{\substack{\chi \\
(\chi \neq \varphi, \chi \neq \psi)}} h_{i_{\varphi} j_{\psi} k_{\chi}}^{\alpha} \omega^{k_{\chi}}, \quad \varphi \neq \psi .
\end{gathered}
$$

Здесь равенство (4.1) получено следующим образом. Если в (3.11) $\lambda_{(\varphi)}^{\alpha} \neq 0$, то, разделив (3.11) на $\lambda_{(\varphi)}^{\alpha}$ и сравнивая полученное равенство с $(3.14)$, получим, что

$$
F_{r}=\left(\lambda_{(\varphi)}^{\alpha}\right)^{-1} h_{r j_{\varphi} j_{\varphi}}^{\alpha}
$$

где правая часть фактически не зависит от $\varphi$. Если теперь (3.12) разделить на $\lambda_{(\varphi)}^{\alpha}$, то получим $(4.1)$.

Из (4.1) следует, что собственные значения $\lambda_{(\varphi)}^{\alpha}$ при всевозможных значениях индексов $\alpha$ и $\varphi$ различаются между собой на постоянные множители.

Из (4.2) следует, что $M^{(1)}$ является вполне омбилическим подмногообразием в $M$. Как мы знаем (лемма 6$), M^{(1)}$ как подмногообразие в $E_{n}$ имеет плоскую нормальную связность и является эйнштейновым. Поскольку $\omega_{r}^{\alpha}=0$, то $T^{(0)}$ как подрасслоение нормального расслоения для $M^{(1)}$ является параллельным, и, следовательно, в нем индуцируется плоская нормальная связность. Тогда нормальные к $M^{(1)}$ векторные поля $e_{r}$ в $T^{(0)}$ можно выбрать так, чтобы они были параллельными в нормальном расслоении подмногообразия $M^{(1)}$. При 
таком выборе полей $e_{r}$ получим $\omega_{r}^{s}=0$ (при $\left.\omega_{r}=0\right)$. Поскольку в общем случае $\omega_{r}^{s}=\Gamma_{r k}^{s} \omega^{k}$, то $\Gamma_{r a}^{s}=0$ и, следовательно, $\omega_{r}^{s}=\Gamma_{r t}^{s} \omega^{t}$.

Дифференцируя внешним образом (4.1) и (4.2) и применяя лемму Картана, получим

$$
\begin{aligned}
& d F_{r}-F_{s} \omega_{r}^{s}=A_{r s} \omega^{s}, \quad A_{r s}=A_{s r} \\
& d F_{r}-F_{s} \omega_{r}^{s}-F_{r} F_{s} \omega^{s}=B_{r} \omega^{j_{\varphi}} .
\end{aligned}
$$

Поскольку левая часть второго равенства не зависит ни от $\varphi$, ни от $j_{\varphi}$, то $B_{r}=0$ и, следовательно, $A_{r s}=F_{r} F_{s}$. Подставляя значение $\omega_{r}^{s}$, окончательно получим

$$
d F_{r}=\left(F_{s} \Gamma_{r t}^{s}+F_{r} F_{t}\right) \omega^{t} .
$$

Из (4.4) следует, что функции $F_{r}$ постоянны на $M^{(1)}$. Так как $e_{r}$ параллельны в нормальном расслоении на $M^{(1)}$, то нормальное к $M^{(1)}$ векторное поле $\xi=F^{r} e_{r}$, где $F^{r}=F_{r}$, также параллельно в нормальном расслоении на $M^{(1)}$. Поэтому, выбирая $e_{m}$ так, что $\xi=\eta e_{m}$, получим $F_{t}=0$ при $t \neq m$ и $F_{m}=\eta$.

Пусть $\eta=0$. Тогда $F_{t}=0$ для любого значения индекса $t$, а из (4.2) следует, что $\omega_{j_{\varphi}}^{r}=0$. Последнее условие означает параллельность распределений $T^{(0)}$ и $T^{(1)}$ на подмногообразии $M$. В силу сопряженности этих распределений относительно второй фундаментальной формы подмногообразия $M$ заключаем, что $M$ локально является прямым произведением их интегральных многообразий, т.е. $M=E_{\mu} \times M^{(1)}$, где $E_{\mu}-$ интегральное многообразие распределения $T^{(0)}$, которое является $\mu$-мерной плоскостью (лемма 4). Следовательно, в этом случае подмногообразие $M$ представляет собой цилиндр над эйнштейновым подмногообразием $M^{(1)}$ (см. замечание 1) с $\mu$-мерными плоскими образующими и является минимальным тогда и только тогда, когда $M^{(1)}$ является минимальным. Вектор средней кривизны $\widetilde{H}$ подмногообразия $M^{(1)}$ определяется, как мы знаем, по формуле (3.22). Так как множество значений индекса $u$ пусто, то, учитывая (3.1), получим

$$
\widetilde{H}=\left(p_{1}+\cdots+p_{q}\right) F^{r} e_{r}=\left(p_{1}+\cdots+p_{q}\right) \eta e_{m}=0 .
$$

Следовательно, $M^{(1)}$ является минимальным. Локальная структура $M^{(1)}$ описывается леммой 7. Поскольку прямое призведение подмногообразий является минимальным тогда и только тогда, когда каждое из подмногообразий минимально, а сферы не являются минимальными подмногообразиями, то $M^{(1)}$ либо представляет собой скрученное произведение сфер одного и того же радиуса (см. лемму 3), либо является прямым произведением минимальных скрученных произведений сфер (одного и того же радиуса). Из (4.1) следует, что при $\eta=0$ собственные значения $\lambda_{(\varphi)}^{\alpha}$ являются постоянными, кроме того, они удовлетворяют следующим условиям (см. (3.1)):

$$
\sum_{\varphi} p_{\varphi} \lambda_{(\varphi)}^{\alpha}=0, \quad \sum_{\varphi}\left(\lambda_{(\varphi)}^{\alpha}\right)^{2}=\rho
$$

Первое из этих условий равносильно, как мы знаем, условию минимальности $M$, а второе - условию его полуэйнштейновости. Подмногообразие $M$, которое в настоящем случае является цилиндром над $M^{(1)}$, определяется следующей пфаффовой системой:

$$
\begin{gathered}
\omega^{\alpha}=0, \quad \omega_{\beta}^{\alpha}=0, \quad \omega_{r}^{\alpha}=0, \quad \omega_{s}^{r}=0, \quad \omega_{j_{\varphi}}^{r}=0, \\
\omega_{i_{\varphi}}^{\alpha}-\lambda_{(\varphi)}^{\alpha} \omega^{i_{\varphi}}=0 .
\end{gathered}
$$


(Ниже, при условии (4.3), мы докажем интегрируемость более общей пфаффовой системы (4.12), из которой система (4.6) получается при $\eta=0$.) Отметим, что, вообе говоря, система (4.6) определяет цилиндр над скрученным произведением сфер (при $p_{\varphi} \geqslant 2$ ). Условия (4.5) на постоянные $\lambda_{(\varphi)}^{\alpha}$ являются необходимыми и достаточными условиями, чтобы этот цилиндр был минимальным и полуэйнштейновым.

Покажем, что постоянные, удовлетворяющие условиям (4.5), всегда существуют. Действительно, пусть в $(n-m)$-мерном евклидовом пространстве $q$-угольник имеет целочисленные стороны $p_{1} \geqslant 2, \ldots, p_{q} \geqslant 2$, где $q \leqslant n-m+1$, и определенную ориентацию. Если последовательно на каждой стороне $q$-угольника отложить по одному единичному вектору, то получим систему векторов $\widetilde{n}_{1}, \ldots, \widetilde{n}_{q}$, которые удовлетворяют условию $p_{1} \widetilde{n}_{1}+\cdots+p_{q} \widetilde{n}_{q}=0$. Умножая это равенство на постоянную $\sqrt{\rho}$, где $\rho>0$, получим систему векторов $\sqrt{\rho} \widetilde{n}_{1}, \ldots, \sqrt{\rho} \widetilde{n}_{q}$, координаты $\widetilde{\lambda}_{(\varphi)}^{\alpha}$ которых удовлетворяют условиям (4.5).

Теперь рассмотрим случай, когда $M^{(1)}$ является прямым произведением минимальных скрученных произведений сфер одного и того же радиуса. В целях ясности, но не умаляя общности, можем предположить, что $q=6$. Пусть распределения

$$
T_{1}=T^{(1,1)}+T^{(1,2)}+T^{(1,3)}, \quad T_{2}=T^{(1,4)}+T^{(1,5)}+T^{(1,6)}
$$

параллельны на $M^{(1)}$ (см. доказательство леммы 7). Тогда $M^{(1)}$ является прямым произведением их интегральных многообразий $M_{1}$ и $M_{2}$, которые являются минимальными скрученными произведениями трех сфер каждое. Параллельность $T_{1}$ и $T_{2}$ аналитически выражается равенством $\omega_{j_{\psi}}^{i_{\varphi}}=0$ при $\varphi=1,2,3$ и $\psi=4,5,6$. Для задания подмногообразия $M$ это уравнение необходимо добавить к системе (4.6). Условие его интегрируемости, как легко показать, имеет следующий вид:

$$
\sum_{\alpha} \lambda_{(\varphi)}^{\alpha} \lambda_{(\psi)}^{\alpha}=0
$$

для тех же значений индексов $\varphi$ и $\psi$. Это условие имеет двоякий геометрический смысл: (а) равенство нулю секционных кривизн между скрученными произведениями $M_{1}$ и $M_{2}$; (б) ортогональность главных векторов кривизны $n_{1}, n_{2}, n_{3}$ к главным векторам кривизны $n_{4}, n_{5}, n_{6}$. Из последнего следует, что $n_{1}+n_{2}+n_{3}=0, n_{4}+n_{5}+n_{6}=0$. Постоянные, удовлетворяющие условиям (4.5) и $(* *)$, всегда существуют. Действительно, чтобы найти такие постоянные, достаточно во взаимно ортогональных подпространствах (размерностей $\geqslant 2)(n-m)$-мерного евклидова пространства построить многоугольники с целочисленными сторонами и векторы, как это было описано выше. Координаты этих векторов и будут удовлетворять указанным условиям.

Пусть $\eta \neq 0$. Отождествляя точку $x \in M^{(1)}$ с радиус-вектором, будем иметь

$$
d\left(x+\frac{1}{\eta} e_{m}\right)=\omega^{a} e_{a}+\frac{1}{\eta} \omega_{m}^{a} e_{a}=\omega^{a} e_{a}+\frac{1}{\eta}\left(-\eta \omega^{a} e_{a}\right)=0 .
$$

Отсюда следует, что

$$
x+\frac{1}{\eta} e_{m}=\text { const }
$$


и, следовательно, подмногообразие $M^{(1)}$ принадлежит некоторой гиперсфеpe $S^{n-1}(\widetilde{R})$ пространства $E_{n}$, для которой вектор $e_{m}$ является нормальным.

Поскольку $\eta e_{m}$ представляет собой вектор средней кривизны для $M^{(1)}$, то $M^{(1)}$ минимально в $S^{n-1}(\widetilde{R})$ (но не минимально в $\left.E_{n}\right)$. Далее, так как $F_{m}=$ $\eta>0$ и $F_{r}=0$ при $r \neq m$, то из (4.4) следует, что $\Gamma_{r t}^{m}=0$. Следовательно, $\omega_{r}^{m}=0$, и так как $\omega_{j_{\varphi}}^{r}=0$ при $r \neq m$ (что следует из (4.2)), то распределение $K$, сопоставляющее каждой точке подмногообразия $M$ линейную оболочку $\mu-1$ векторов $e_{r}, r \neq m$, является параллельным на $M$ и вполне интегрируемым. Его интегральное многообразие представляет собой $(\mu-1)$-мерную плоскость, которую обозначим через $E_{\mu-1}$. Ортогональное дополнение к $K_{x}$ в касательном пространстве $T_{x}(M)$ имеет вид

$$
T_{x}^{(1)}+L_{x}=T_{x}^{(1,1)}+\cdots+T_{x}^{(1, q)}+L_{x}
$$

где $L_{x}-$ прямая с направляющим вектором $e_{m}$. Соответствующее распределение $T^{(1)}+L$ также параллельно на $M$ и вполне интегрируемо. Его интегральное многообразие будем обозначать через $\widetilde{M}$. В силу сопряженности распределений $K$ и $T^{(1)}+L$ относительно второй фундаментальной формы подмногообразия $M$ заключаем, что $M$ локально является прямым произведением их интегральных многообразий, т.е. $M=E_{\mu-1} \times \widetilde{M}$. Здесь $\widetilde{M}$ представляет собой $\left(p_{1}+\cdots+p_{q}+1\right)$-мерное полуэйнштейново подмногообразие некоторого $(n-\mu+1)$-мерного евклидова пространства.

Покажем, что $\widetilde{M}$ является конусом над $M^{(1)}$. Действительно, поскольку $d e_{m}=-\eta \omega^{a} e_{a}$, то $e_{m}=$ const при $\omega^{a}=0$. Отсюда следует, что $\widetilde{M}$ является либо конусом (с исключенной точечной вершиной, ибо в ней теряется регулярность), либо цилиндром над подмногообразием $M^{(1)}$ с образующей $L_{x}$ в каждой точке. Поскольку $\eta \neq 0$, то $\widetilde{M}$ может быть только конусом. Далее, так как $E_{\mu-1}$ является плоскостью, то в качестве локальных координат на $E_{\mu-1}$ можем взять декартовы прямоугольные координаты. Тогда $\omega_{s}^{r}=0$ для всех значений индексов и, следовательно, $\Gamma_{s t}^{r}=0$ для любых значений индексов $r, s, t$. На подмногообразии $\widetilde{M}$ интегральное многообразие распределения $L$ есть прямая с направляющим вектором $e_{m}$. Обозначим через $x^{m}$ координату на этой прямой. Так как $d \omega^{m}=0$ (что легко проверить), то можем считать, что $\omega^{m}=d x^{m}$. Если в (4.4) $r=m$, то получается уравнение $d \eta=\eta^{2} d x^{m}$. Интегрируя его, находим

$$
\eta=\frac{1}{C-x^{m}}
$$

где $C$ - постоянная интегрирования. Подстановка в (4.1) приводит к формуле

$$
\lambda_{(\varphi)}^{\alpha}=\frac{C_{\varphi}^{\alpha}}{C-x^{m}},
$$

где $C_{\varphi}^{\alpha}-$ постоянные интегрирования. На основании (3.15) и (3.1) получаем следующие соотношения:

$$
\begin{gathered}
\rho=\frac{\sum_{\alpha}\left(C_{\varphi}^{\alpha}\right)^{2}}{\left(C-x^{m}\right)^{2}}, \\
\sum_{\alpha}\left(C_{\varphi}^{\alpha}\right)^{2}=\sum_{\alpha}\left(C_{\psi}^{\alpha}\right)^{2}, \quad \varphi \neq \psi, \\
\sum_{\varphi} p_{\varphi} C_{\varphi}^{\alpha}=0 .
\end{gathered}
$$


Постоянные $C_{\varphi}^{\alpha}$, удовлетворяющие условиям (4.10) и (4.11), можно выбирать так же, как и постоянные, удовлетворяющие условиям (4.5), как это было описано выше. Вычисляя эйнштейнову константу подмногообразия $M^{(1)}$ по формуле (3.23), получим

$$
\widetilde{\rho}=\widetilde{\rho}_{a}=\rho-\left(p_{1}+\cdots+p_{q}-1\right) \eta^{2}=\frac{\sum_{\alpha}\left(C_{(\varphi)}^{\alpha}\right)^{2}-\left(p_{1}+\cdots+p_{q}-1\right)}{\left(C-x^{m}\right)^{2}} .
$$

Из этой формулы следует, что путем выбора постоянных $C_{\varphi}^{\alpha}$ величину $\widetilde{\rho}$ можно сделать как равной нулю, так и отличной от нуля. Так как $x^{m}$ является координатой на образующей конуса, то вдоль образующей $\widetilde{\rho}$ изменяется, а на $M^{(1)}$, когда $x^{m}$ фиксировано, $\widetilde{\rho}$ действительно является постоянной. Из формул (4.7)-(4.9) следует, что $\eta, \lambda_{(\varphi)}^{\alpha}, \rho$ также изменяются вдоль образующей конуса.

Итак, пусть числа $C_{\varphi}^{\alpha}$ удовлетворяют условиям (4.10) и $(4.11)$, и пусть $\eta$ и $\lambda_{(\varphi)}^{\alpha}$ определяются по формулам (4.7) и (4.8). В евклидовом пространстве $E_{n}$ рассмотрим следующую дифференциальную систему:

$$
\left\{\begin{array}{l}
\omega^{\alpha}=0, \quad \omega_{\beta}^{\alpha}=0, \quad \omega_{r}^{\alpha}=0, \quad \omega_{s}^{r}=0, \quad \omega_{j_{\varphi}}^{r}=0, \quad r \neq m, \\
\omega_{i_{\varphi}}^{\alpha}-\lambda_{(\varphi)}^{\alpha} \omega^{i_{\varphi}}=0, \quad d \ln \left|\lambda_{(\varphi)}^{\alpha}\right|-\eta \omega^{m}=0, \quad d \eta-\eta^{2} \omega^{m}=0, \quad \omega_{j_{\varphi}}^{m}-\eta \omega^{j_{\varphi}}=0 .
\end{array}\right.
$$

Путем непосредственного вычисления внешних дифференциалов левых частей уравнений этой системы можем убедиться в том, что они обращаются в нуль в силу уравнений самой системы. Наиболее трудно проверить это для шестого уравнения, для которого вычисления проведем непосредственно. Вопервых, отметим, что по предположению выполняется равенство (4.3). Во-вторых, докажем, что имеет место равенство

$$
\sum_{\substack{\psi \\(\psi \neq \varphi)}}\left(\lambda_{(\psi)}^{\alpha}-\lambda_{(\varphi)}^{\alpha}\right) \omega_{j_{\psi}}^{i_{\varphi}} \wedge \omega^{j_{\psi}}=0
$$

для фиксированного субиндекса $\varphi$. Действительно, на основании (4.3) имеем

$$
\sum_{\substack{\psi \\(\psi \neq \varphi)}} \sum_{\substack{\chi \\(\chi \neq \varphi, \chi \neq \psi)}} h_{i_{\varphi} j_{\psi} k_{\chi}} \omega^{k_{\chi}} \wedge \omega^{j \psi}=0
$$

Здесь знак равенства написан на основании того, что $h_{i_{\varphi} j_{\psi} k_{\chi}}^{\alpha}$ симметричны по индексам $j_{\psi}$ и $k_{\chi}$, а выражение $\omega^{k_{\chi}} \wedge \omega^{j_{\psi}}$ кососимметрично по этим индексам, кроме того, можем считать, что субиндексы $\psi$ и $\chi$ пробегают одно и то же множество значений, т.е. $\chi$ может принимать значение $\psi$, так как

$$
h_{i_{\varphi} j_{\psi} j_{\psi}}=0
$$

при $\varphi \neq \psi$ (лемма 1). Из (4.13) следует, что

$$
\sum_{\substack{\psi \\(\psi \neq \varphi)}} \lambda_{(\psi)}^{\alpha} \omega_{j_{\psi}}^{i_{\varphi}} \wedge \omega^{j_{\psi}}=\lambda_{(\varphi)}^{\alpha} \sum_{\substack{\psi \\(\psi \neq \varphi)}} \omega_{j_{\psi}}^{i_{\varphi}} \wedge \omega^{j_{\psi}}
$$


Если теперь вычислить внешние дифференциалы выражений $\omega_{i_{\varphi}}^{\alpha}$ и $\lambda_{(\varphi)}^{\alpha} \omega^{i_{\varphi}}$, то получим

$$
\begin{array}{r}
d \omega_{i_{\varphi}}^{\alpha}=-\lambda_{(\varphi)}^{\alpha} \omega_{j_{\varphi}}^{i_{\varphi}} \wedge \omega^{j_{\varphi}}-\sum_{\substack{\psi \\
(\psi \neq \varphi)}} \lambda_{(\psi)}^{\alpha} \omega_{j_{\psi}}^{i_{\varphi}} \wedge \omega^{j_{\psi}}, \\
d\left(\lambda_{(\varphi)}^{\alpha} \omega^{i_{\varphi}}\right)=-\lambda_{(\varphi)}^{\alpha} \omega_{j_{\varphi}}^{i_{\varphi}} \wedge \omega^{j_{\varphi}}-\lambda_{(\varphi)}^{\alpha} \sum_{\substack{\psi \\
(\psi \neq \varphi)}} \omega_{j_{\psi}}^{i_{\varphi}} \wedge \omega^{j_{\psi}} .
\end{array}
$$

Теперь из (4.14) следует, что $d\left(\omega_{i_{\varphi}}^{\alpha}-\lambda_{(\varphi)}^{\alpha} \omega^{i_{\varphi}}\right)=0$.

Таким образом, система (4.12) вполне интегрируема. Она фактически и задает описанное выше нормально плоское минимальное полуэйнштейново подмногообразие $M=E_{\mu-1} \times \widetilde{M}$ с интегрируемым распределением кодефектности. Отметим, что при $\eta=0$ система (4.12) обращается в систему (4.6) и определяет цилиндр $E_{\mu} \times M^{(1)}$. Теорема 1 доказана.

В случае, когда $\eta \neq 0$, радиусы сфер $S^{p_{\varphi}}(R)$ и гиперсферы $S^{n-1}(\widetilde{R})$ определяются соответственно равенствами $R^{2}=\left(\rho+\eta^{2}\right)^{-1}, \widetilde{R}^{2}=\eta^{-2}$. Следовательно,

$$
R=\frac{c-x^{m}}{\sqrt{\sum_{\alpha}\left(C_{\varphi}^{\alpha}\right)^{2}+1}}, \quad \widetilde{R}=C-x^{m}
$$

и радиусы $R$ и $\widetilde{R}$ являются линейными, но непостоянными функциями на $L$.

\section{§5. О классификации Ric-полусимметрических гиперповерхностей}

В теореме 1 одним из условий является условие интегрируемости распределения кодефектности подмногообразия. В настоящем параграфе в качестве примера, когда распределение кодефектности не предполагается интегрируемым, мы рассмотрим Ric-полусимметрические гиперповерхности в $E_{n}$.

Классификация Ric-полусимметрических гиперповерхностей в $E_{n}$ была дана автором в [6] (под названием Ric-полупараллельных гиперповерхностей). Там была доказана следующая классификационная

Теорема 2. В евклидовом пространстве $E_{m+1}$ гиперповерхность $M$ удовлетворяет условию $R(X, Y) \cdot R_{1}=0$ тогда и толъко тогда, когда она является открытой частью одного из следующих обгектов:

(1) гиперсферы $S^{m}$ в $E_{m+1}$,

(2) гиперконуса вращения $C^{m}$ в $E_{m+1}$,

(3) произведения $S^{n} \times E_{m-n}$, где $S^{n}-$ гиперсфера в $E_{n+1}, a E_{m-n}-(m-n)$ мерная плоскость $, n=2, \ldots, m-1$,

(4) произведения $C^{n} \times E_{m-n}$, где $C^{n}-$ гиперконус вращения в $E_{n+1}, a E_{m-n}-$ $(m-n)$-мерная плоскость $, n=2, \ldots, m=1$,

(5) гиперповерхности ранга $\leqslant 2$,

(6) полуэйнштейновой гиперповерхности в $E_{m+1}, m \geqslant 5$, которая несет трехкомпонентную ортогональную сопряженную систему, состоящую из двух сфер, $S^{p}\left(r_{1}\right), p \geqslant 2, u S^{q}\left(r_{2}\right), q \geqslant 2, u$ прямой $L, u$ представляет собой конус с одномерными плоскими образующими (прямая $L$ в качестве образующей в каждом точке) над прямым произведением $S^{p}\left(r_{1}\right) \times S^{q}\left(r_{2}\right)$, 
которое является эйнштейновым подмногообразием в $E_{m+1}$ и принадлежит гиперсфере $S^{m}(r) \subset E_{m+1}$, әде радиусы $r_{1}, r_{2}, r$ связаны условием $r^{2}=r_{1}^{2}+r_{2}^{2}$ и являются линейными (не постоянными) функциями на L,

(7) произведения $K^{n} \times E_{m-n}$, где $K^{n}$ - полуэйнштейнова гиперповерхность в $E_{n+1}$, описываемая, как и $K^{m}$, в $n$. (6), а $E_{m-n}-(m-n)$-мерная плоскость, $n=5, \ldots, m-1$.

В этой теореме полуэйнштейнова гиперповерхность (6) была получена фактически при неявном предположении интегрируемости распределения кодефектности гиперповерхности. Другие возможные случаи не были рассмотрены. Здесь мы покажем, что все остальные случаи приводят только к противоречию. Следовательно, формулировка классификационной теоремы 2 не меняется. Изложение будем вести с использованием обозначений, принятых в работе [6].

Поскольку $M$ является гиперповерхностью, то имеется только одна матрица $\left\|h_{i j}^{m+1}\right\|$ второй фундаментальной формы (в дальнейшем индекс $m+1$ будем опускать). Пусть матрица $\left\|h_{i j}\right\|$ имеет два ненулевых собственных значения $\lambda$ и $\mu$ кратностей $p \geqslant 2$ и $q \geqslant 2$ соответственно и нулевое собственное значение кратности $m-p-q$. Тогда $\mu=a-\lambda=(p-1) \lambda /(1-q)$, где $a-$ средняя кривизна (подробности см. в [6]). Обозначим через $T_{x}^{(\lambda)}, T_{x}^{(\mu)}, T_{x}^{(0)}$ подпространства собственных векторов матрицы $\left\|h_{i j}\right\|$ в касательном пространстве $T_{x}(M)$. Здесь $T_{x}^{(0)}$ фактически является пространством дефектности гиперповерхности в точке $x$. Через $T^{(\lambda)}, T^{(\mu)}, T^{(0)}$ будем обозначать соответствующие распределения. Индексы будут пробегать следующие значения:

$$
b, c=1, \ldots, p, \quad u, v=p+1, \ldots, p+q, \quad s, t=p+q+1, \ldots, m .
$$

Будем предполагать также, что адаптированный к $M$ ортонормированный репер $\left(e_{1}, \ldots, e_{m}, e_{m+1}\right)$ согласован со структурой подпространств $T_{x}^{(\lambda)}, T_{x}^{(\mu)}, T_{x}^{(0)}$. Это значит, что $e_{b} \in T_{x}^{(\lambda)}, e_{u} \in T_{x}^{(\mu)}, e_{s} \in T_{x}^{(0)}$. Тогда равенство (3.3) для настоящего случая будет иметь вид

$$
\left(d \lambda_{i}\right) \delta_{i j}+\left(\lambda_{j}-\lambda_{i}\right) \omega_{j}^{i}=h_{i j k} \omega^{k},
$$

где в правой части нет суммирования по $i$. Придавая индексам различные значения, получим следующую систему:

$$
\left\{\begin{array}{l}
d \lambda=h_{s} \omega^{s}, \\
\mu \omega_{u}^{s}=h_{s u b} \omega^{b}+\frac{p-1}{1-q} h_{s} \omega^{u}, \\
\lambda \omega_{b}^{s}=h_{s} \omega^{b}+h_{s b u} \omega^{u}, \\
(\mu-\lambda) \omega_{b}^{u}=h_{s b u} \omega^{s},
\end{array}\right.
$$

где $h_{s}=h_{b b s}$. (Эта система в [6] фигурирует под номером (4.5).) Дифференцируя первое равенство в (5.1) внешним образом, получим

$$
\left(d h_{s}-h_{r} \omega_{s}^{r}\right) \wedge \omega^{s}+\left(\frac{1}{\lambda}-\frac{1}{\mu}\right) h_{s} h_{b u}^{s} \omega^{b} \wedge \omega^{u}=0 .
$$

Отсюда легко получаются следующие соотношения:

$$
\begin{gathered}
d h_{s}-h_{r} \omega_{s}^{r}=A_{s_{t}} \omega^{t}, \quad A_{s_{t}}=A_{t s}, \\
\sum_{s} h_{s} h_{s u b}=0 .
\end{gathered}
$$


В плоскости $T_{x}^{(0)}$ рассмотрим векторы

$$
F=\sum_{s} h_{s} e_{s}, \quad F_{b u}=\sum_{s} h_{b u s} e_{s}
$$

Условие (5.3) фактически является условием ортогональности инвариантного вектора $F$ к системе векторов $\left(F_{b u}\right)$. Очевидно, что $0 \leqslant \operatorname{rang}\left(F_{b u}\right) \leqslant \operatorname{dim} T_{x}^{(0)}$. Рассмотрим все возможные случаи. Пусть $\operatorname{rang}\left(F_{b u}\right)=0$. Тогда $h_{\text {bus }}=0$ для всех значений индексов. Этот случай подробно рассмотрен в [6]. Он приводит к гиперповерхностям из пунктов (6) и (7) теоремы 2 . Пусть теперь $\operatorname{rang}\left(F_{b u}\right)=\operatorname{dim} T_{x}^{(0)}$. Так как вектор $F$ принадлежит $T_{x}^{(0)}$ по определению и ортогонален к $T_{x}^{(0)}$, то $F=0$. Следовательно, $h_{s}=0$ для любого значения индекса $s$. В этом случае система (5.1) принимает следующий вид:

$$
\left\{\begin{array}{l}
\lambda=\text { const }, \quad \mu=\frac{p-1}{1-q} \lambda=\text { const } \\
\lambda \omega_{b}^{s}=h_{s b u} \omega^{u} \\
\mu \omega_{u}^{s}=h_{s u b} \omega^{b} \\
(\mu-\lambda) \omega_{u}^{b}=h_{b u s} \omega^{s} .
\end{array}\right.
$$

Дифференцируя внешним образом второе уравнение системы (5.4), придем к следующим соотношениям:

$$
\begin{gathered}
d h_{s b u}-h_{s b v} \omega_{u}^{v}-h_{t b u} \omega_{s}^{t}-h_{s c u} \omega_{b}^{c}=h_{s b u v} \omega^{v}, \\
h_{b u t} h_{s c}^{u}+h_{s b u} h_{t c}^{u}=0 .
\end{gathered}
$$

Поскольку последнее равенство имеет место для всех значений индексов, то, полагая в нем $c=b, t=s$, получим $h_{s b u} \cdot h_{s b}^{u}=0$. Следовательно, $h_{b u s}=0$, и тогда $\omega_{b}^{s}=\omega_{u}^{s}=\omega_{u}^{b}=0$. Получается прямое произведение двух сфер и плоскости, которое не может быть гиперповерхностью. Рассмотрим теперь случай, когда $0<\operatorname{rang}\left(F_{b u}\right)<\operatorname{dim} T_{x}^{(0)}$. В этом случае в плоскости $T_{x}^{(0)}$ можем провести дополнительную адаптацию векторов $e_{s}$. Именно, вектор $e_{m}$ можем выбрать так, чтобы он был коллинеарен инвариантному вектору $F$. Тогда $F=h_{m} e_{m}$ и, следовательно, $h_{s}=0$ при $s \neq m$. Будем считать, что $h_{m} \neq 0$ (иначе получим второй случай). Условие (5.3) теперь принимает вид $h_{m} h_{m u b}=0$. Тогда $h_{m u b}=0$ и система (5.1) приводится к виду

$$
\left\{\begin{array}{l}
d \lambda=h_{m} \omega^{m}, \\
\mu \omega_{b}^{m}=\frac{p-1}{1-q} h_{m} \omega_{u}, \\
\lambda \omega_{b}^{m}=h_{m} \omega^{b}, \\
(\mu-\lambda) \omega_{u}^{b}=h_{u b s} \omega^{s}, \\
\mu \omega_{u}^{s}=h_{s u b} \omega^{b}, \quad s \neq m, \\
\lambda \omega_{b}^{s}=h_{s b u} \omega^{u}, \quad s \neq m .
\end{array}\right.
$$

Рассмотрим теперь уравнение (5.2) и, полагая в нем $s=m$, получим

$$
d h_{m}=A_{m t} \omega^{t} .
$$


Если в (5.2) $s \neq m$, то будем иметь

$$
\omega_{s}^{m}=-\frac{A_{s t}}{h_{m}} \omega^{t}
$$

Далее, поскольку плоскость $T_{x}^{(0)}$ является евклидовым пространством, то, не умаляя общности, можем считать, что в $T_{x}^{(0)}$ в качестве локальных координат выбраны прямоугольные декартовы координаты. Тогда $\omega_{s}^{t}=0$ при $\omega^{m+1}=0$, $\omega^{b}=0, \omega^{u}=0$ (последняя система задает распределение $\left.T^{(0)}\right)$. Отсюда следует, что

$$
\omega_{s}^{t}=\Gamma_{s u}^{t} \omega^{u}+\Gamma_{s b}^{t} \omega^{b} .
$$

Следовательно, в (5.7) $A_{s t}=0$, где $s \neq m$, а индекс $t$ принимает все свои значения. Полагая $t=m$, получим $A_{m s}=0, s \neq m$. С учетом (5.6) будем иметь

$$
\omega_{s}^{m}=0, \quad d h_{m}=A_{m m} \omega^{m} .
$$

Подставляя во второе уравнение системы $(5.5) \mu=(p-1) \lambda /(1-q)$ и дифференцируя его внешним образом, а также учитывая остальные уравнения этой системы, получим соотношение

$$
\left(d h_{m}+\frac{2}{\lambda} h_{m}^{2} \omega^{u}\right) \wedge \omega^{m}+\left(\frac{\lambda}{\mu} h_{t u c} \omega_{m}^{t}-\frac{1}{\mu} h_{m} h_{u c t} \omega^{t}\right) \wedge \omega^{c}=0,
$$

где $t \neq m$. Отсюда в силу (5.8) имеем

$$
\frac{2}{\lambda} h_{m}^{2} \omega^{u} \wedge \omega^{m}+\frac{1}{\mu} h_{m} h_{u c t} \omega^{c} \wedge \omega^{t}=0, \quad t \neq m .
$$

Поскольку 2-формы $\omega^{u} \wedge \omega^{m}, \omega^{c} \wedge \omega^{t}$ независимы, то $h_{m}=0$, что приводит к противоречию.

Итак, все возможные случаи рассмотрены. Только условие $h_{b u s}=0$, которое, как мы знаем, равносильно интегрируемости распределения кодефектности гиперповерхности, приводит к содержательным результатам.

В связи с проведенными в этом параграфе исследованиями возникает следующий вопрос: можно ли в теореме 1 освободиться от условия интегрируемости распределения кодефектности?

\section{Список литературы}

[1] В. А. Мирзоян, "Ric-полусимметрические подмногообразия", Итоги науки и техники. Проблемы геометрии, 23, ВИНИТИ, М., 1991, 29-66; англ. пер.: V. A. Mirzoyan, "Ric-semisymmetric submanifolds", J. Math. Sci., 70:2 (1994), 1624-1646.

[2] В. А. Мирзоян, "Структурные теоремы для римановых Ric-полусимметрических пространств", Изв. вузов. Матем., 6 (1992), 80-89; англ. пер.: V. A. Mirzoyan, "Structure theorems for Riemannian Ricci semi-symmetric spaces", Russian Math. (Iz. VUZ), 36:6 (1992), 75-83.

[3] В. А. Мирзоян, "Конусы над эйнштейновыми пространствами", Изв. НАН Армении. Матем., 33:5 (1998), 46-54; англ. пер.: V. A. Mirzoyan, "Cones over Einstein spaces", J. Contemp. Math. Anal., 33:5 (1998), 40-46. 
[4] В. А. Мирзоян, "Скрещенные произведения, конусы над эйнштейновыми пространствами и классификация одного класса Ric-полупараллельных подмногообразий”, Изв. РАН. Сер. матем., 67:5 (2003), 107-124; англ. пер.: V. A. Mirzoyan, "Warped products, cones over Einstein spaces, and classification of Ric-semiparallel submanifolds of a certain class", Izv. Math., 67:5 (2003), 955-973.

[5] E. Boeckx, O. Kowalski, L. Vanhecke, Riemannian manifolds of conullity two, World Sci. Publ., Singapore-River Edge, NJ, 1996.

[6] В. А. Мирзоян, "Классификация Ric-полупараллельных гиперповерхностей в евклидовых пространствах", Матем. сб., 191:9 (2000), 65-80; англ. пер.: V. A. Mirzoyan, "Classification of Ric-semiparallel hypersurfaces in Euclidean spaces", $S b$. Math., 191:9 (2000), 1323-1338.

[7] F. Defever, "Ricci-semisymmetric hypersurfaces", Balkan J. Geom. Appl., 5:1 (2000), 81-91.

[8] Ю. Г. Лумисте, "Полусимметрические подмногообразия", Итоги науки и техники. Проблемы геометрии, 23, ВИНИТИ, М., 1991, 3-28; англ. пер.: Yu. G. Lumiste, "Semisymmetric submanifolds", J. Math. Sci., 70:2 (1994), 1609-1623.

[9] F. Dillen, S. Nölker, "Semi-parallelity, multi-rotation surfaces and the helix-property", J. Reine Angew. Math., 435 (1993), 33-63.

[10] S. Nölker, "Isometric immersions of warped products", Differential Geom. Appl., 6:1 (1996), 1-30.

[11] Ü. Lumiste, "Semi-parallel submanifolds as some immersed fibre bundles with flat connections", Geometry and topology of submanifolds, VIII, Proceedings of the international meeting on geometry of submanifolds (Brussels, Belgium and Nordfjordeid, Norway, 1995), World Sci. Publ., Singapore-River Edge, NJ, 1996, 236-244.

[12] D. Perrone, "Contact Riemannian manifolds satisfying $R(X, \xi) \cdot R=0$ ", Yokohama Math. J., 39:2 (1992), 141-149.

[13] B. J. Papantoniou, "Contact Riemannian manifolds satisfying $R(X, \xi) \cdot R=0$ and $\xi \in(k, \mu)$-nullity distribution", Yokohama Math. J., 40:2 (1993), 149-161.

[14] F. Defever, R. Deszcz, L. Verstraelen, "On pseudosymmetric para-Kähler manifolds", Colloq. Math., 74:2 (1997), 253-260.

[15] M. Dąbrowska, F. Defever, R. Deszcz, D. Kowalczyk, "Semisymmetry and Ricci-semisymmetry for hypersurfaces of semi-Euclidean spaces", Publ. Inst. Math. (Beograd) (N.S.), 67 (2000), 103-111.

[16] Ü. Lumiste, "Normally flat semiparallel submanifolds in space forms as immersed semisymmetric Riemannian manifolds", Comment. Math. Univ. Carolin., 43:2 (2002), 243-260.

[17] Ü. Lumiste, "Semiparallel isometric immersions of 3-dimensional semisymmetric Riemannian manifolds", Czechoslovak Math. J., 53:3 (2003), 707-734.

[18] M. M. Tripathi, J.-S. Kim, "On the concircular curvature tensor of a $(\kappa, \mu)$-manifold", Balkan J. Geom. Appl., 9:1 (2004), 104-114.

[19] П.К. Рашевский, Риманова геометрия и тензорный анализ, 2-е изд., Наука, M., 1964; нем. пер. 1-го изд.: Р. K. Raschewski, Riemannsche Geometrie und Tensoranalysis, VEB Deutscher, Berlin, 1959.

[20] Б. А. Дубровин, С. П. Новиков, А. Т. Фоменко, Современная геометрия. Методы и приложения, Наука, М., 1986; англ. пер.: В. A. Dubrovin, A. Т. Fomenko, S. P. Novikov, Modern geometry - methods and applications. Part I. The geometry of surfaces, transformation groups, and fields, Grad. Texts in Math., 93, Springer-Verlag, New York, 1992.

[21] М.М. Постников, Лекиии по геометрии. Семестр V. Риманова геометрия, Факториал, М., 1998; англ. пер.: М. M. Postnikov, Geometry VI. Riemannian geometry, Encyclopaedia Math. Sci., 91, Springer-Verlag, Berlin, 2001. 
[22] Ш. Кобаяси, К. Номидзу, Основы дифференииальной геометрии, т. II, Наука, М., 1981; пер. с англ.: Sh. Kobayashi, K. Nomizu, Foundations of differential geometry, vol. II, Intersci. Publ., New York-London-Sydney, 1969.

[23] B. Chen, Geometry of submanifolds, Pure Appl. Math., 22, Marcel Dekker, New York, 1973.

[24] S.S. Chern, W.H. Chen, K.S. Lam, Lectures on differential geometry, Ser. Univ. Math., 1, World Sci. Publ., Singapore-River Edge, NJ, 1999.

[25] А. Т. Фоменко, Вариационнъе методы в топологии, Наука, М., 1982.

[26] Дао Чонг Тхи, А. Т. Фоменко, Минималънъе поверхности и проблема Плато, Наука, M., 1987; англ. пер.: Dào Trong Thi, A. T. Fomenko, Minimal surfaces, stratified multivarifolds, and the Plateau problem, Transl. Math. Monogr., 84, Amer. Math. Soc., Providence, RI, 1991.

[27] Sh.-Sh. Chern, N. H. Kuiper, "Some theorems on the isometric imbedding of compact Riemann manifolds in Euclidean space", Ann. of Math. (2), 56:3 (1952), 422-430.

[28] В.А. Мирзоян, "Структурные теоремы для Ric-полусимметрических подмногообразий и геометрическое описание одного класса минимальных полуэйнштейновых подмногообразий", Матем. сб., 197:7 (2006), 47-76; англ. пер.: V.A. Mirzoyan, "Structure theorems for Ricci-semisymmetric submanifolds and geometric description of a class of minimal semi-Einstein submanifolds", Sb. Math., 197:7 (2006), 997-1024.

[29] В.А. Мирзоян, "Подмногообразия с коммутирующим нормальным векторным полем", Итоги науки и техники. Проблемы геометрии, 14, ВИНИТИ, М., 1983, 73-100; англ. пер.: V. A. Mirzoyan, "Submanifolds with commuting normal vector field", J. Soviet Math., 28:2 (1985), 192-207.

[30] J. D. Moore, "Isometric immersions of Riemannian products", J. Differential Geometry, 5 (1971), 159-168.

\section{В. А. Мирзоян (V. А. Mirzoyan)}

Государственный инженерный университет Армении, г. Ереван;

Московский государственный университет сервиса, Ереванский филиал

E-mail: mirzoyan_vanya@front.ru
Поступила в редакцию 16.10.2006 и 07.11.2007 